20 1020

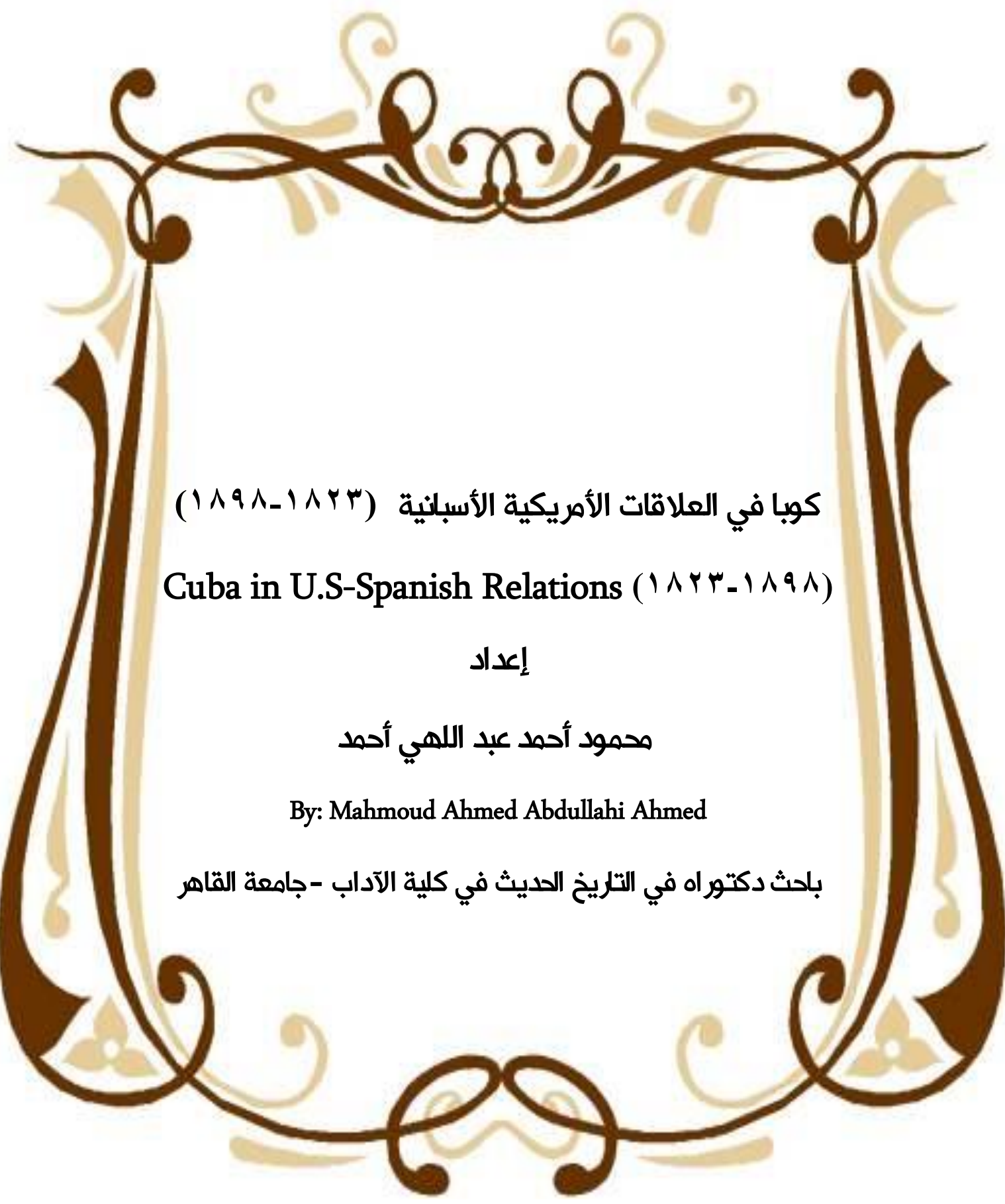


(1) (1)

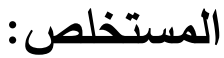

أصبحت كوبا محور العلاقات الأمريكية -الأسبانية، إذ دخلت هذه المنطقة في

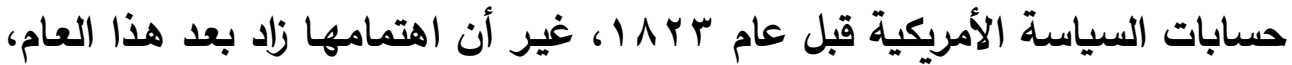

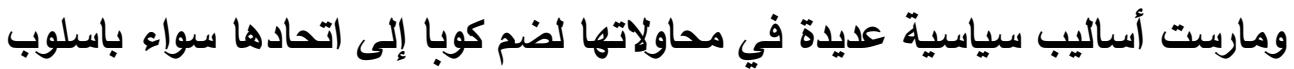

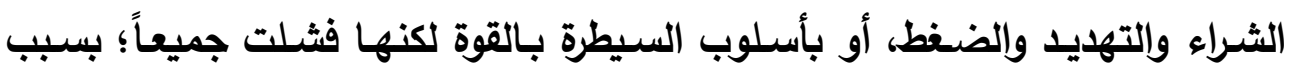

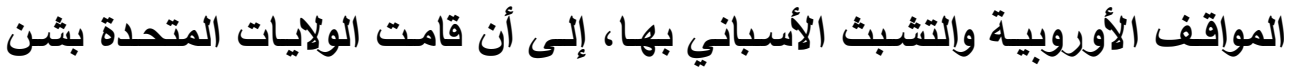

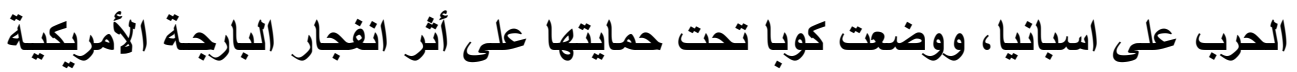

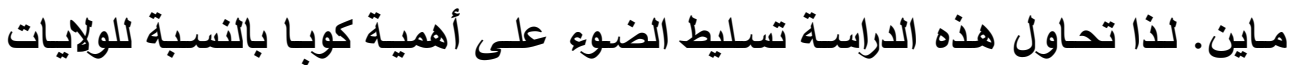

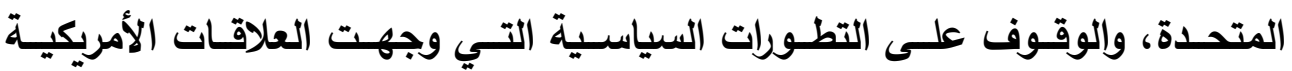

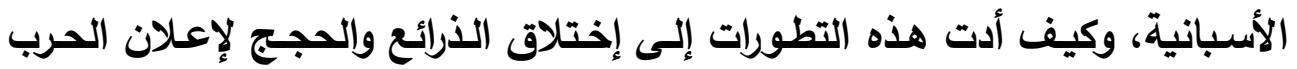

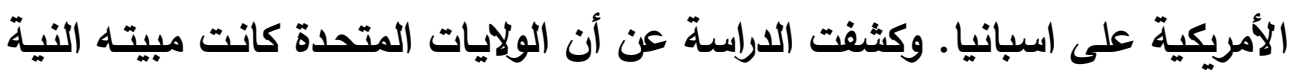

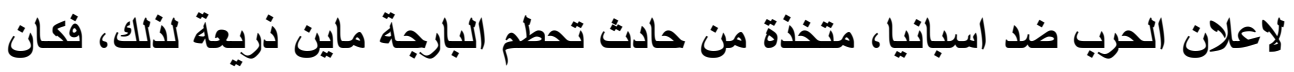

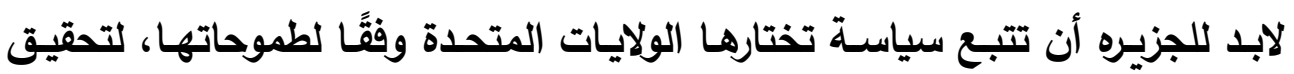

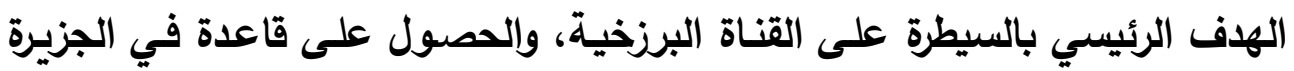
تؤمن المحافظة على القناة بعد افتتاحها.

الكلمــات المفتاحيـة: القنــاة البرزخيـة- مبــأ مـونرو - حـرب السـنوات العشـر فيرجينوس - البارجة ماين - الصحافة الصفراء. 
أدى تــهور الأوضـاع العامـة في اسبانيا خـلال القرن التاسـع عشر نتيجـة

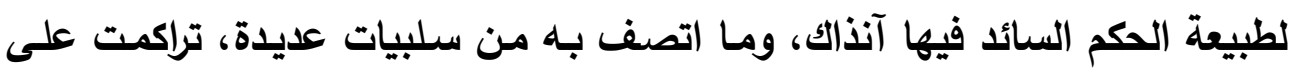

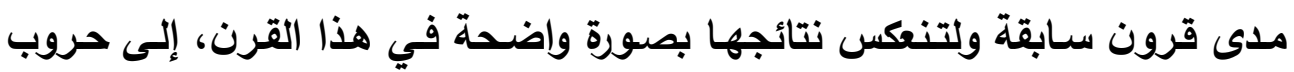

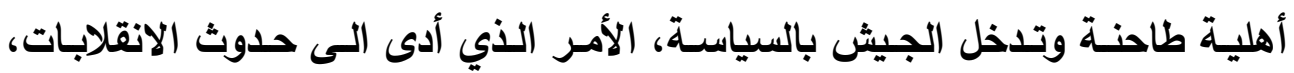

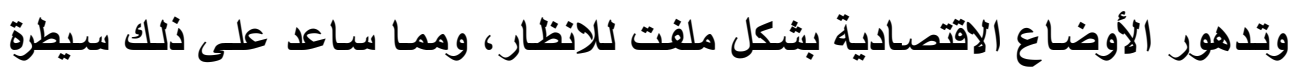

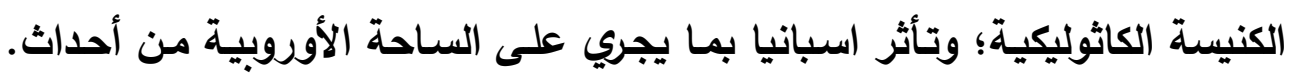

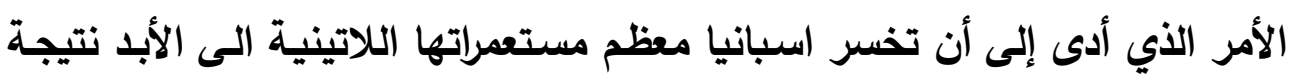
مباشرة لما شهدتة اسبانيا.

وعلى الصعيد الأخر شـهات الولايـات المتحدة في ذات الفترة أوضـاعاً تكاد

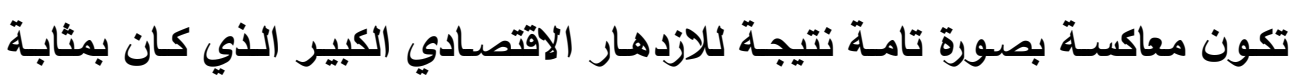

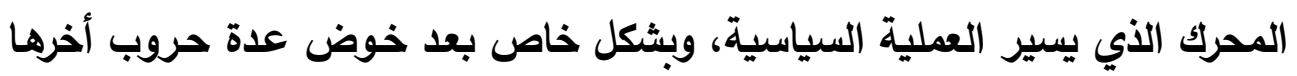

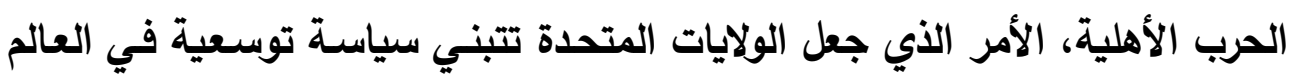

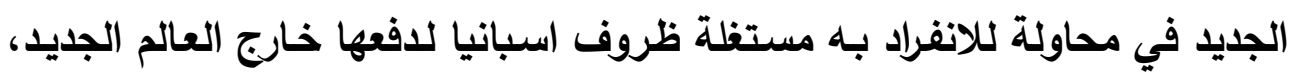

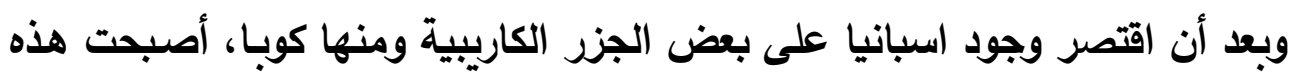

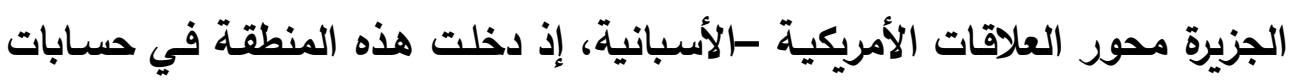

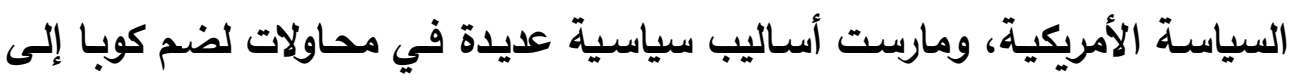

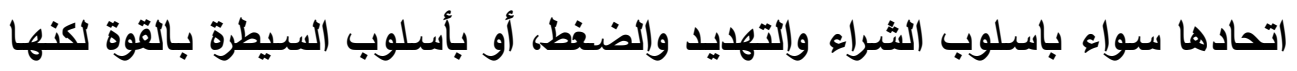

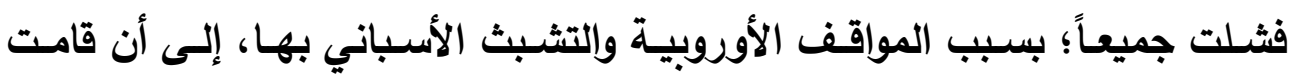

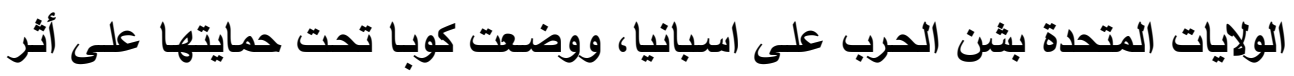
انفجار البارجة الأمريكية ماين.

\footnotetext{
مجلة كلية الأداب- جامعة بني سويف
} 


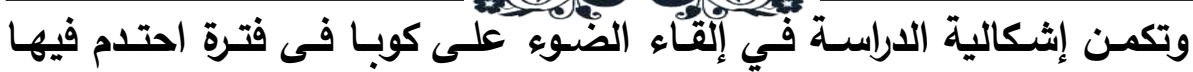

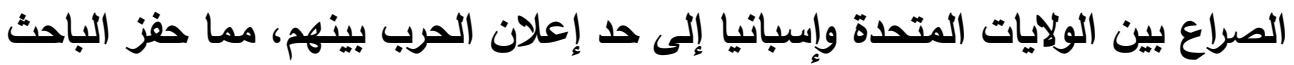

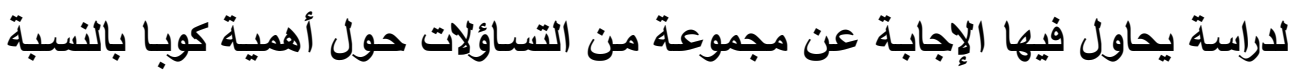

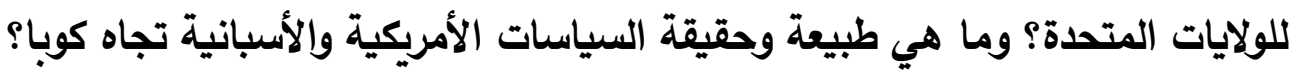

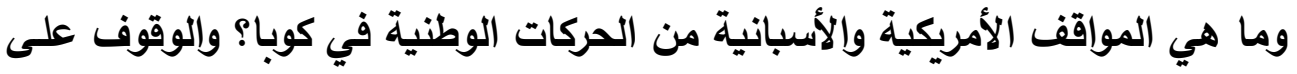

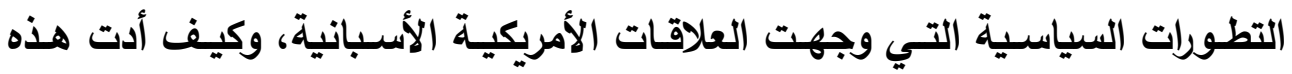

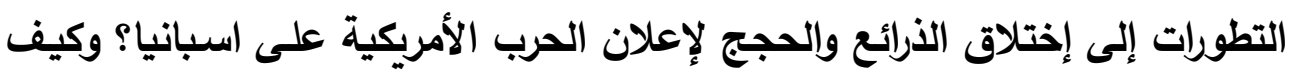
كانت ردود الفعل الأسبانية على تلك التطورات؟

إن الهـف الـرئيس مسن هذه الدراسـة هـو عرض وتحليل تـاريخ العلاقـات

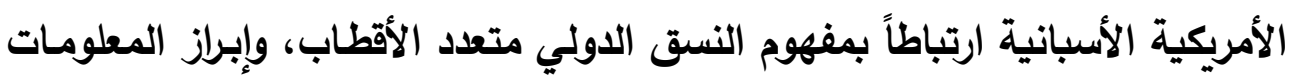

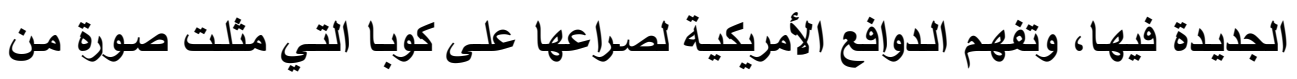

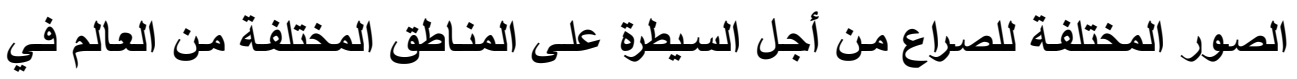

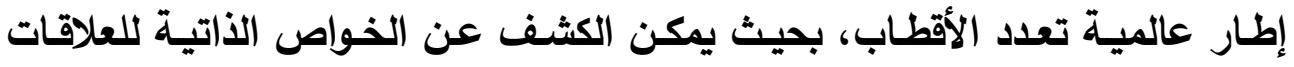

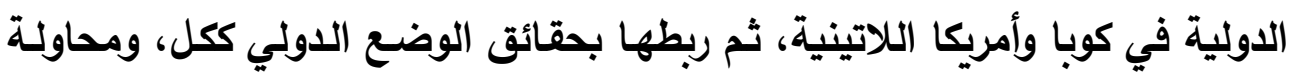

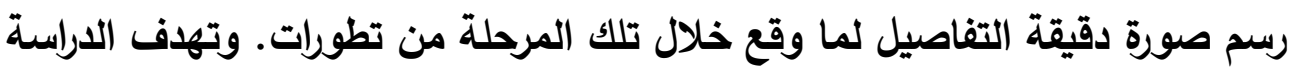
أيضاً الى عرض الأفعال وردود الأفعال المتبادلة بين الدولتين في كوبـا من ناحية،

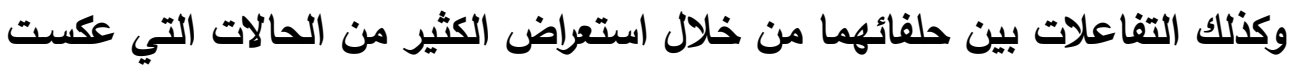
هذا التنافس المحتلم من ناحية أخرى.

أمّا منهج الاراسة فقد استخدمت المنهج الموضوعي من حيث تقسيم الدراسـة

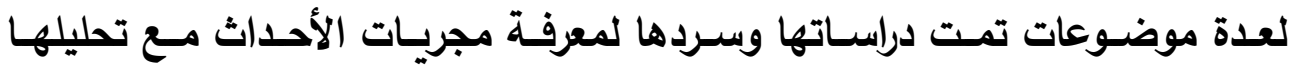

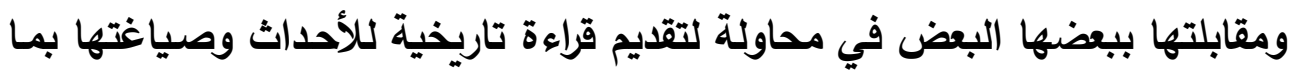

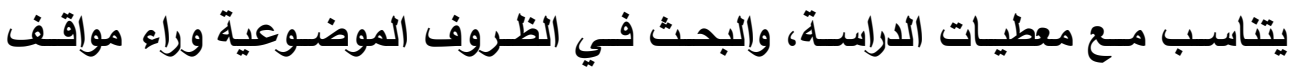




\section{(1) (1)}

الأطـراف المعنيـة، ملتزمـاً بوحدة الموضـوع في السـياق التـاريخي العـام، وعرض لكأفعال وردود الأفعال بين الأطراف المعنية بالدراسة.

وجاءت دراستي في محورين رئيسيين، فضـلاً عن المقدمـة والخاتمـة، وقائمـة بالمصادر وإلمراجع التي اعتمدت عليها الدراسة، وذلك على النحو التالي:

المحور الأول: اهتمامات ومصالح الولايات المتحدة الأمريكية في كوبا:

ترجـ بدايـة اهتمامـات الولايـات المتحدة الأمريكية الأولحى بجزيـرة كوبـا, إلى

عهد جورج واشنطن عندما تطلع للسيطرة على هذه الجزيرة لتصبح قاعدة للتوسـع ومد النفوذ الأمريكي إلى داخل أمريكا الوسطى والجنوبية، فضلاً عن تحكمها في أحـد مـــلي القنـاة البرزخيـة التـي أرادت الولايـات المتحـدة حفرهـا عبـر أمريكـا

الوسطى (')

وزاد هذا الاهتمام في عامی • ^ 1، حيث نظر إليها الرئيس الأمريكي تومـاس جيفرسون "(Thomas Jefferson" على إنها أعظم إضـافة ذات فائدة يجعلها ضمن النطاق الأمريكي"() وظهر ذلك في تصريحاته حيث صرح قائلاً "في الوقت

(r) Sherry, Michael S., The Democratic Experience: A short American history (New York, 19^•),P. ${ }^{r r}$.

توماس جيفرسون: هو الرئيس الثالث للولايات المتحدة الأمريكية، تولى الرئاسة من ا. ـ1 ا-

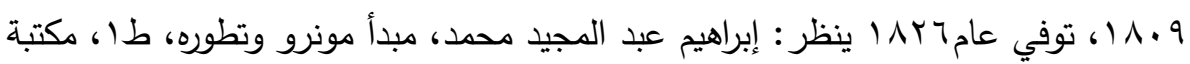

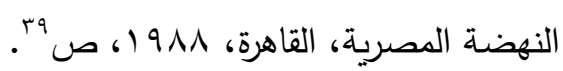

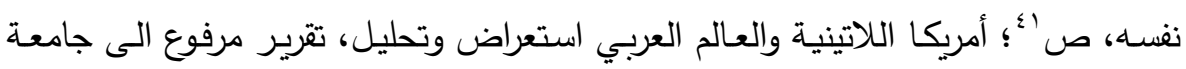

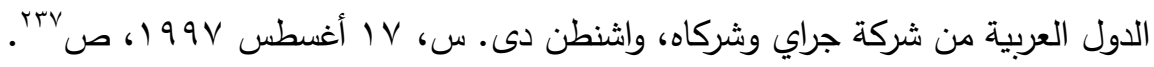

مجلة كلية الأداب- جامعة بني سويف


الأي يتعين على الولايات المتحدة الأمريكية أستعادة فلوريدا من الفرنسيين....لابد لها من الحصول على جزيرة كوبا بطبيعية الحال، لأنها تكاد تفدو جزعاً من البلاد.

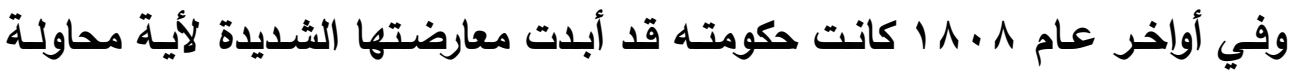

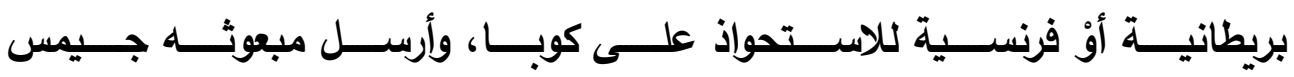
وليكنسون"James Wilkinson" إلى كوبا, للتفاوض مع الحكومة الاسبانية حول

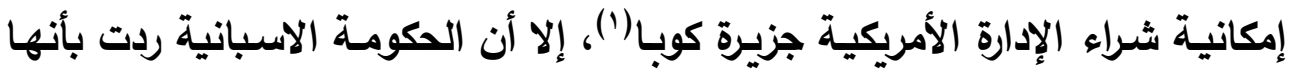

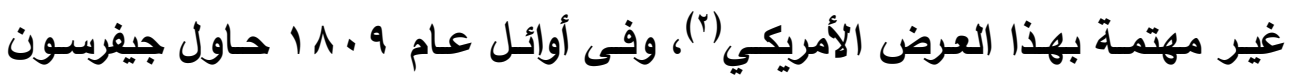

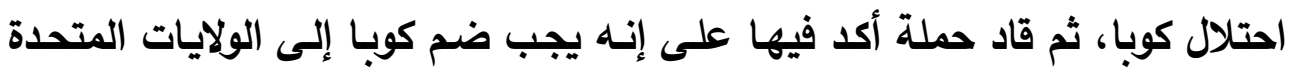

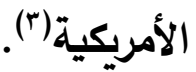

وبعـد أن تـولى جيمس ماديسـون (") رئاســة الإدارة

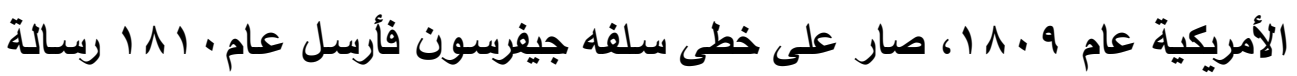

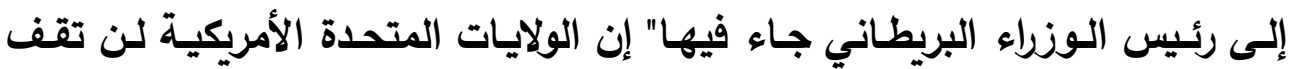

$$
\text { للمزيد حول المحاولات الأمريكية لضم كوبا سواء بالثراء أو السيطرة انظر : }
$$

Chapman, Charles E.; A history of the Cuban Republic. A study in Hispanic American politics., New York, Octagon Books, 1979,Vol.I.; p.p. ${ }^{\{\wedge-\imath 0}$.

(") Fontain, Roger W, on Negotiation with Cuba U.S.A American Enterprise institute for Public policy, Research, U.S.A, I qvo,p.r; Franklin, Jane,Cuba and The United States, Chronological History ,sixth Printing, New York , ץ... ฯ, P. ;

سهير جبر ، الولايات التتحدة وأمريكا اللاتينية ضمن كتاب الإمبراطورية الأمريكية، ج)، القاهرة،

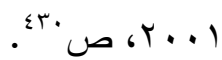

(") Fontaine, Roger W., OP.Cit., PP ${ }^{4-\uparrow}$.

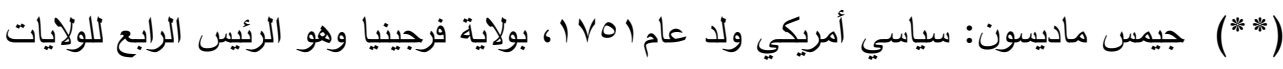

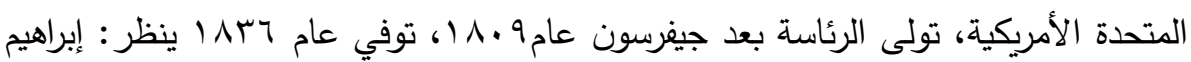

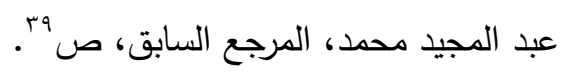

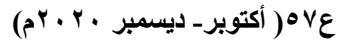
مجلة كلية الأداب- جامعة بني سويف 


\section{0.}

مكتوفة الأيدي، إذا ما كانت هنالك أي محاولة بريطانية للسيطرة على كوبا"(')، خوفاً

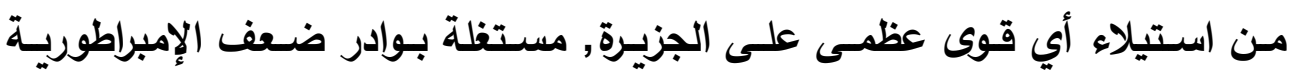

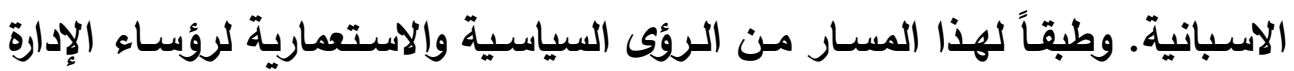

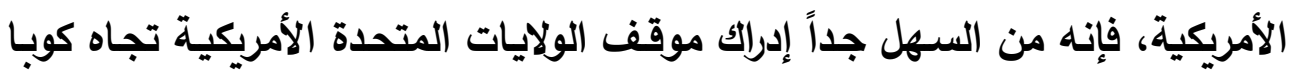

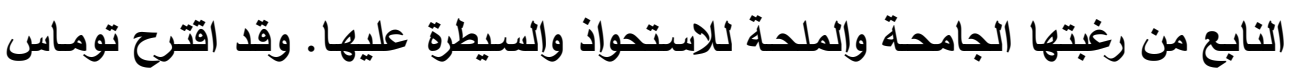
جيفرسـون علي الرئيس ماديسون أن يعرض علـي الامبراطور الفرنسي نـابليون

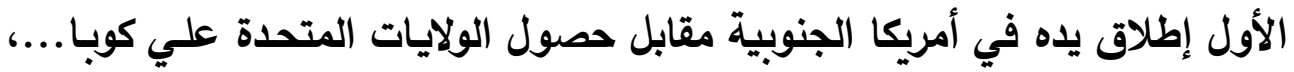

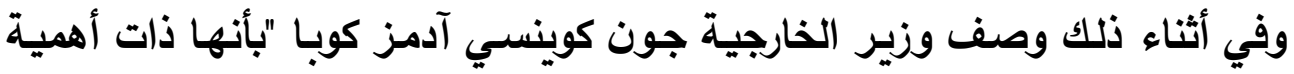

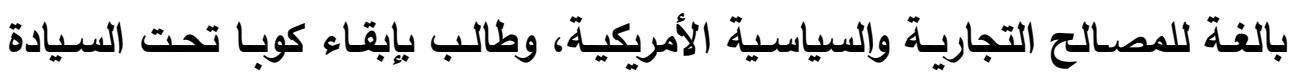
الإسبانية إلي أن تقع في أيدي الولايات المتحدة بثكل أو بآخر (†).

ولــم يمسض وقت طويـل حتـى رضـت السـلطات الإسـبانية لرغبـات الإدارة

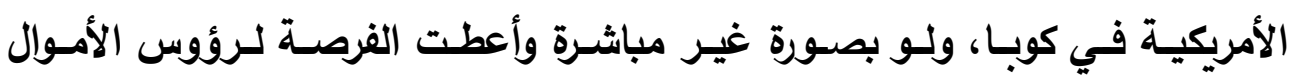

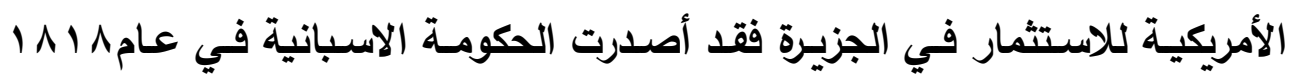

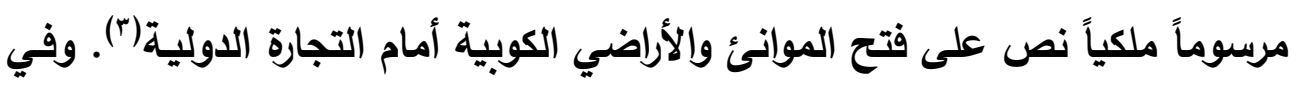

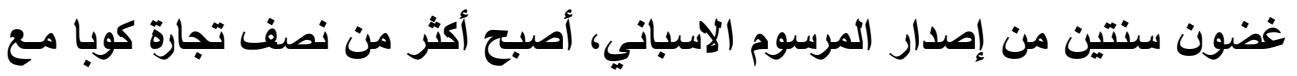
الولايات المتحدة الأمريكية. لذا أثار هذا التطور في الاتصالات بين الإدارة الأمريكية الإنية

(') Franklin, Jane, OP. Cit., P."

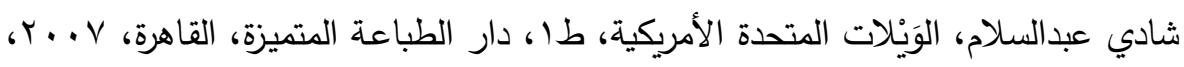
$\therefore$

(') Robinson, Albert G.,Cuba old and New, Tower of Lafuerza,Havana, 1919 ,p.p. ${ }^{\wedge \&-\wedge \uparrow}$. 


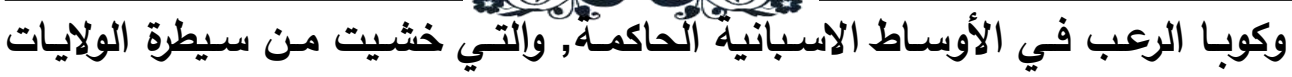

المتحدة الأمريكية على الجزيرة (')

ولعل أبـز مـا يؤكد هذه المخـاوف رسـالة وزيـر الخارجيـة الأمريكي جون

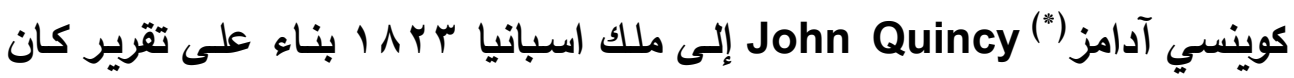

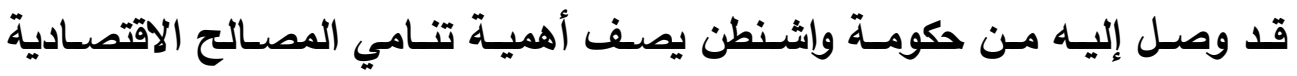

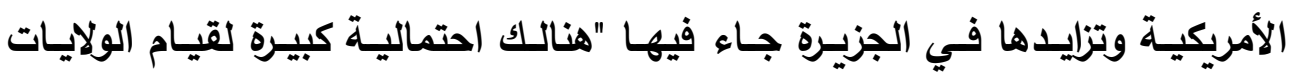

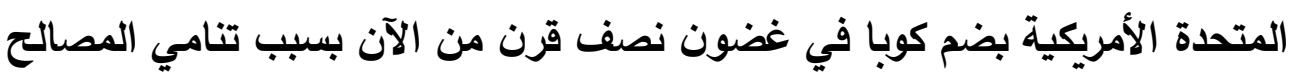

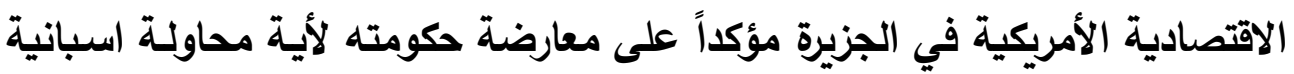

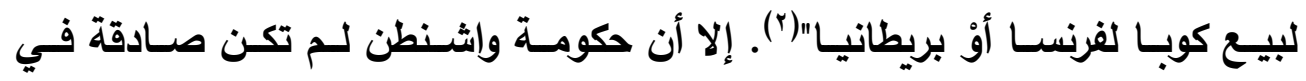
رسالتها، ولم تمهل الحكومة الاسبانية مدة نصف قرن لضم الجزيرة.

وانطلاقاً من هذه السياسـة أخذت الولايات المتحدة الأمريكية تراقب أوضاع الجزيرة عن كثب باحثة عن الفرصة المناسبة لتحقيق هدفها بضمها إليها، وقد

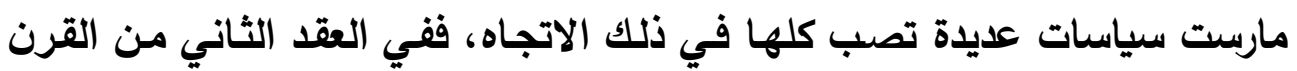

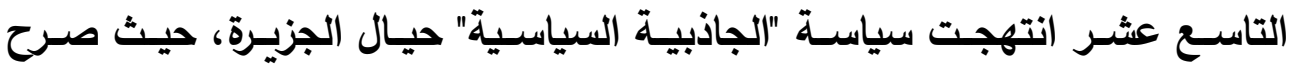

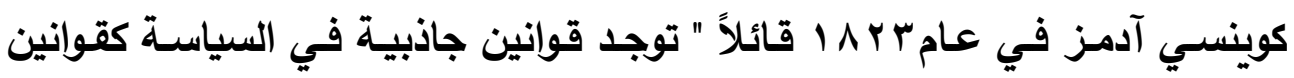

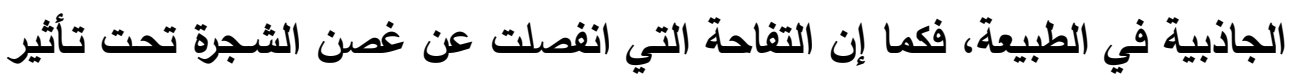

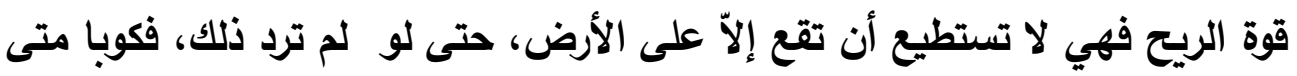

(") Christopher, Abel and Torrents, Nissa, Eds Jose Marti: Revolutionary Democrat, London, 1914 , PP. ${ }^{\text {r. - - r.a }}$.

جون كوينسي أدامز : من أعظم وزراء خارجية الولايات المتحدة في تاريخ الاتحاد الفيدرالي

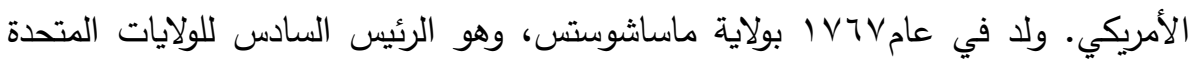

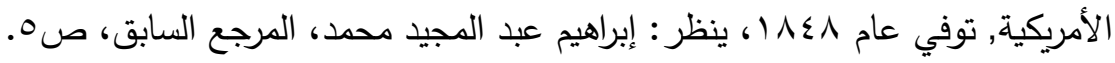

(₹) Bailey, Thomas A., OP.Cit.,PP. 
مـا قطعت الصلة التي تربطها باسبانيا فعليها بالضرورة أن تنجذب نحو أمريكا

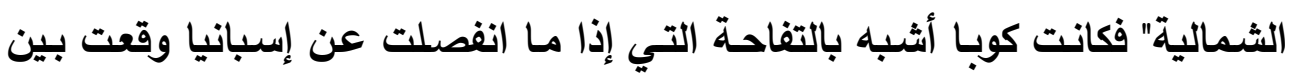

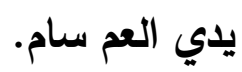

كانت كوبـا أشبه بجسيم يجذبه مغنـاطيس، حيث تؤكد هذه الرسـالة الهدف

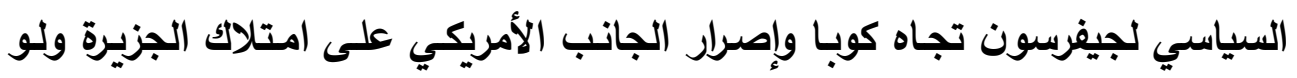

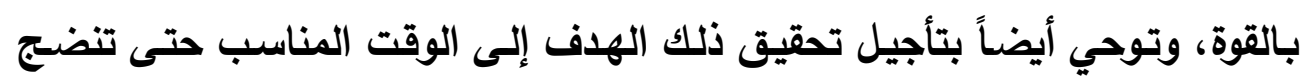

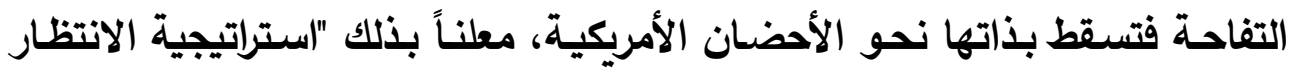

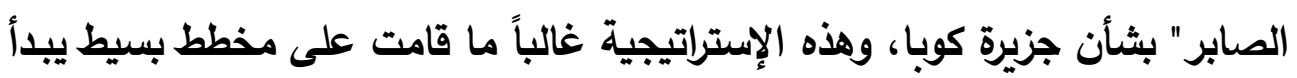

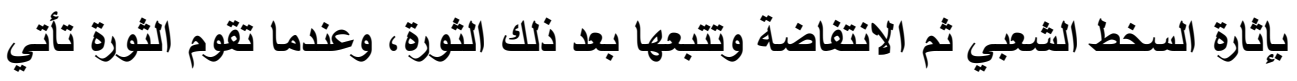

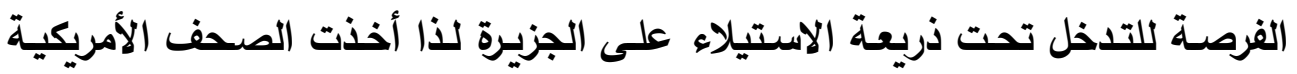

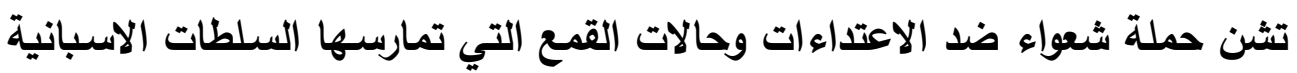

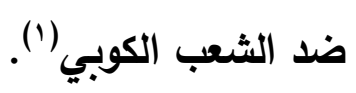

ولم تمض سوى أثهر على تلك الرسالة حتى أعلن الرئيس الأمريكي جيمس

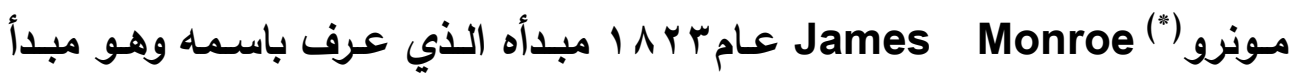

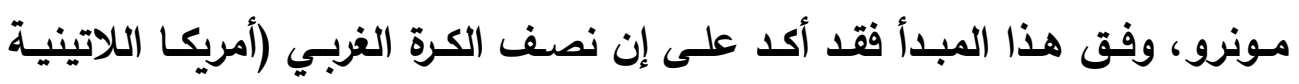

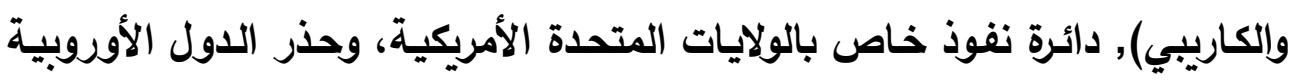

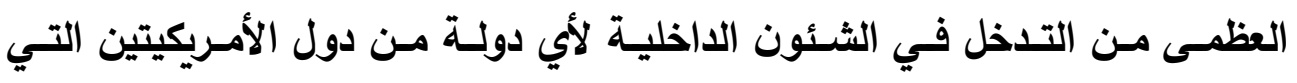

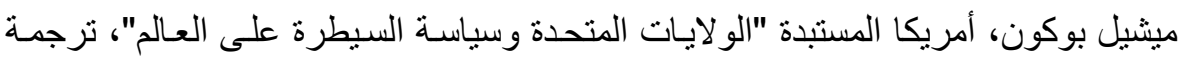

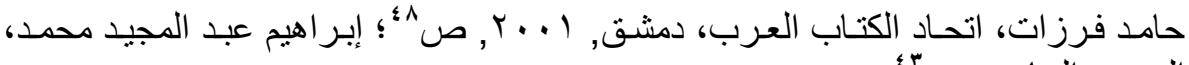

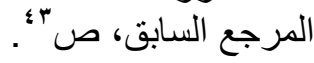

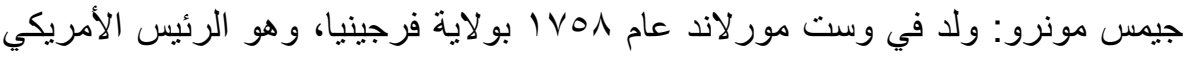

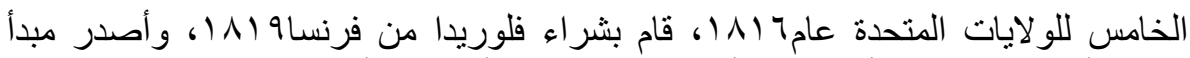

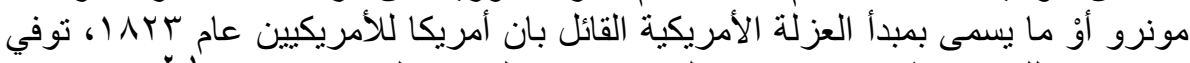

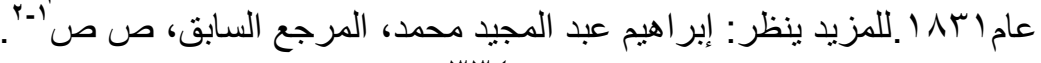

مجلة كلية الأداب- جامعة بني سويف 
حصلت على استقلالها مؤخراً. وأقامتت الولايتات أمتحدة في عدد كبير من دول

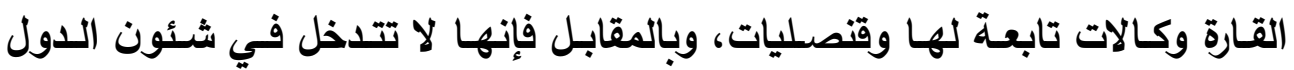
الأوروبية")

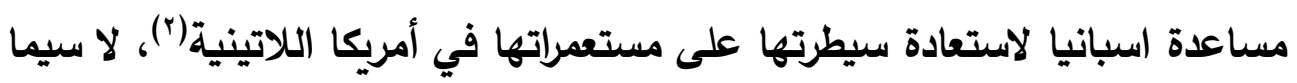

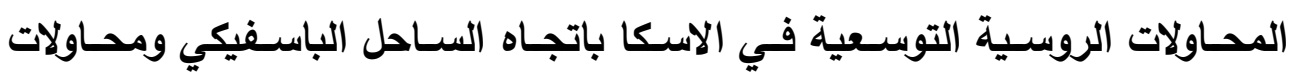

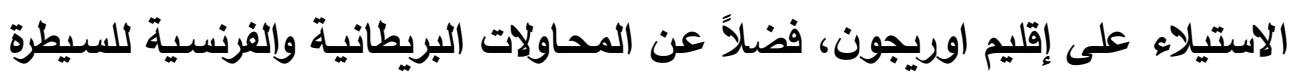

على المستعمرات الاسبانية بسبب ضعف الأخيرة (").

وقبل إصدار هذا المبدأ اقترح وزير خارجية بريطانيا "جورج كانتج" على نظيره

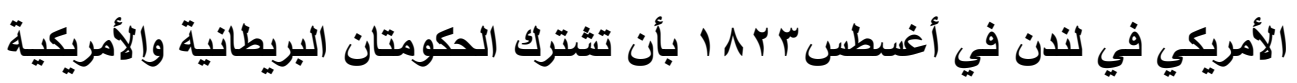

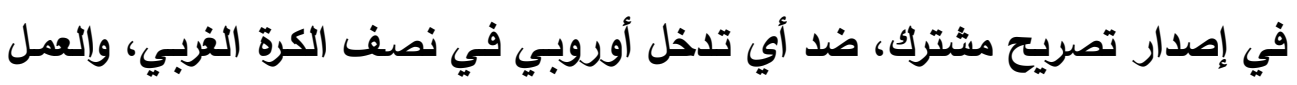

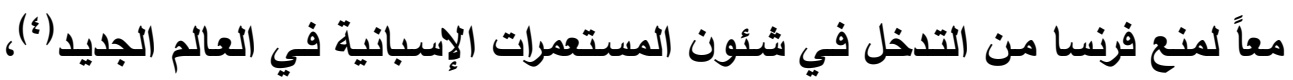

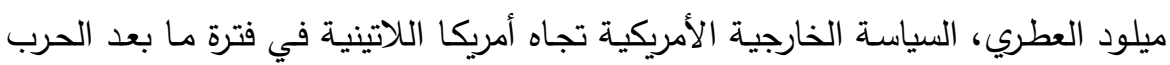
الباردة، رسالة ماجستير ، قسم العلوم السياسية، فرع العلاقات الدولية والدراسات الإستراتيجية،

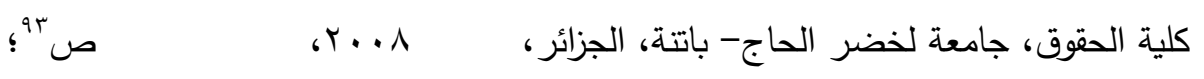
Franklin, Jane, OP. Cit. , P. •.

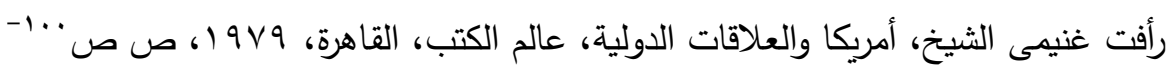

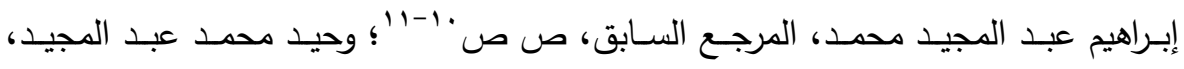

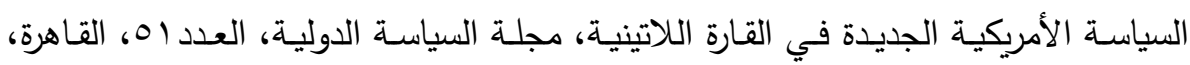

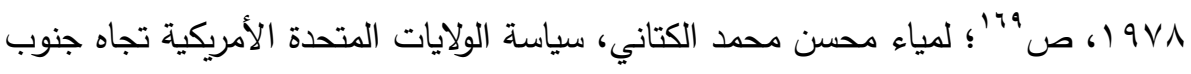

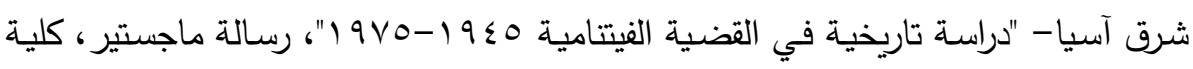

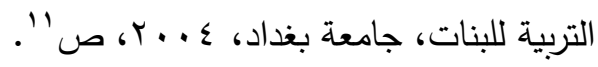
السيد رجب حراز ، مبدأ مونرو وأزمـة التضـامن الأمريكي، مجلة السية السياسية الدولية، السنة

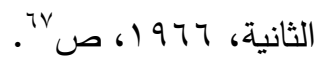




\section{(1) (1)}

وبذلك يكون مبدأ مونرو هو في الأساس اقتراح مشترك من قبل دولتين اتفقتا على إصدار هذا التصريح للحد من النفوذ الفرنسي.

جاء الإعلان عن مبأ مونرو ليضع الطموحات الاستعمارية للولايات المتحدة الأمريكية موضع التففيذ، ولاسيما في نصف العالم الغربي بشكل عام وكوبـا بثكل خاص. إذ عد مبدأ مونرو النقطة الرئيسة في التحول الاستعماري الأمريكي على مولى الرغم من أن بداية هذه الطموحات اتسمت بالطابع الدفاعي الموجه ضد أي تدخل أوروبي في شئون الأمريكيتين، إلا أنها بمرور الوقت أصبحت ذات طابع هجومي

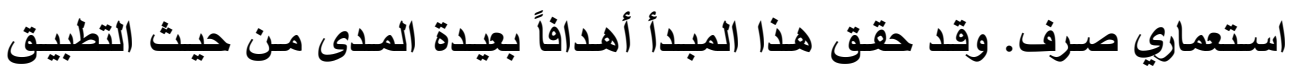
الفعلي، إذ رفع الرئيس مـونرو شـعاره "أمريكا للأمريكيين" ومـن خـلال هذا الثـعار يتجلى بوضوح الهدف الأمريكي الاستعماري لبسط نفوذه على القارة اللاتينية متذرعاً بالحفاظ على المصالح الأمريكية.

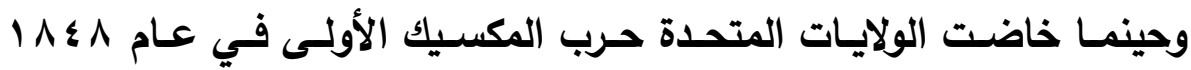
مقتطعة نصـف مسـاحة المكسيك بعدها، أكد الجنـرال جرانـ Grant على وحدة مصير الأمريكيتين مطالباً بحق التذخل في القارة بأكملها، وقد ترجم هذا إلى تخطيط الأمريكيين للاستيلاء على كوبـا(')، وعندما نشبت ثورة العبيد في العام ذاته كان مرشتح الحزب الايمقراطي لمنصب رئيس الولايات المتحدة الأمريكية السيناتور لويس

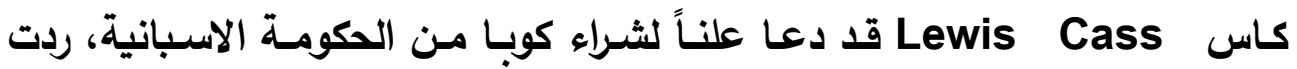
الحكومة الاسبانية بالموافقة المبدئية على بيع كوبا لها. مما جعل الإدارة الأمريكية ترحب برد السلطات الإسبانية وأعلنت أنها على الاستعداد لدفع مـا يصل إلى مئسة

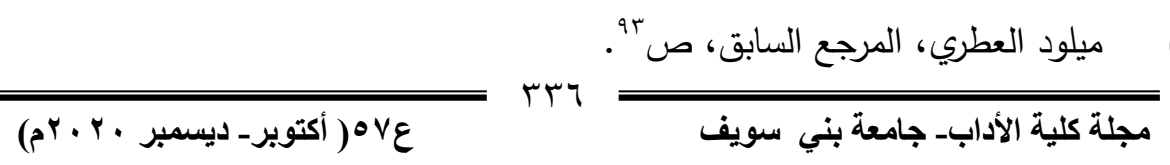




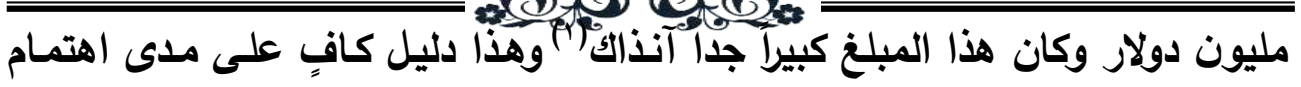

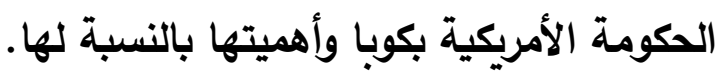

عقدات المفاوضات بين المسئولين الأمريكيين ونظرائهم الإسبان عام 9 ؟1 لكن هذه المفاوضات سرعان ما انهارت، بسبب الموقف الانجليزي الفرنسي المؤيد

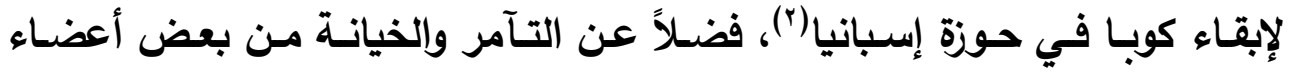

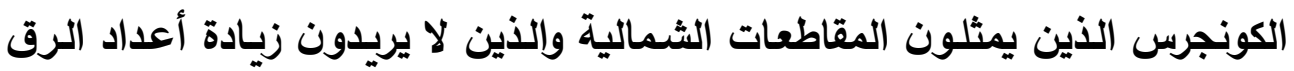
والعبيد في الولايات المتحدة الأمريكية بضم كوبا التي كانت تحوي على التى أعداد كبيرة

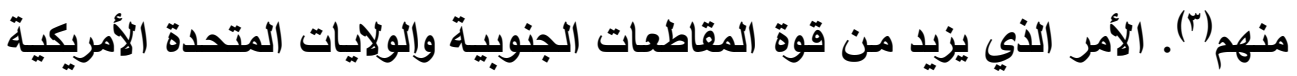

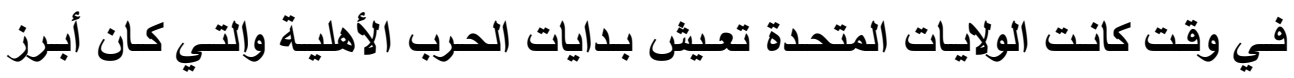
أسبابها سياسـة التمييز العنصري. ورغم ذلك اتجهت الإدارة الأمريكية إلى المجال التهال

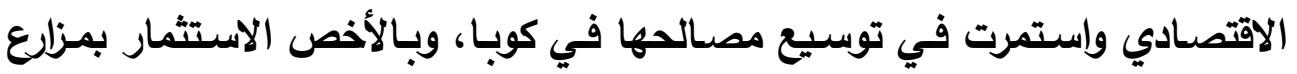

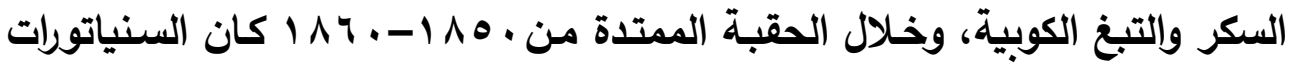

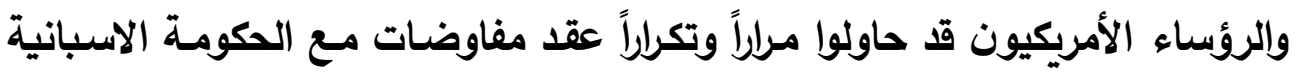

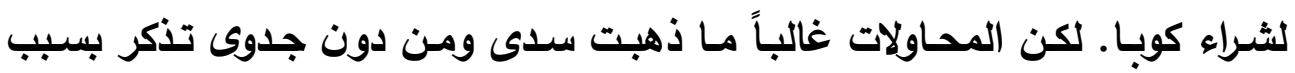

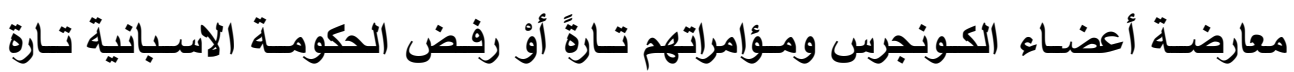

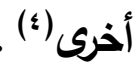

(") Bailey, Thomas A, OP, Cit., PP. ${ }^{\curlyvee \wedge-r \wedge \wedge}$; رأفت الشيخ غنيمي، أمريكا

$$
\text { والعالم، صن }
$$

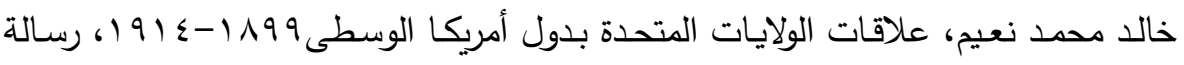

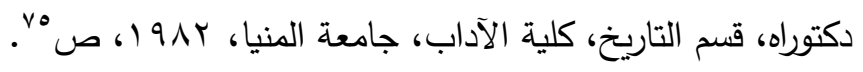

( ${ }^{\circ}$ ado, Roberto Regal, OP.Cit., PP ${ }^{r+r v}$.

(') Franklin, OP, Cit., P. ${ }^{\circ}$; Almaflor, Ada, Under the Royal plans: Childhoods in Cuba, London, $19 \wedge \wedge, \mathrm{P}^{\wedge 0}$. 


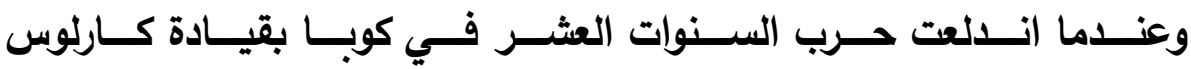
مانويـل(") Marlos Mael de Cespedes ضـــ السـلطات الاسـبانية

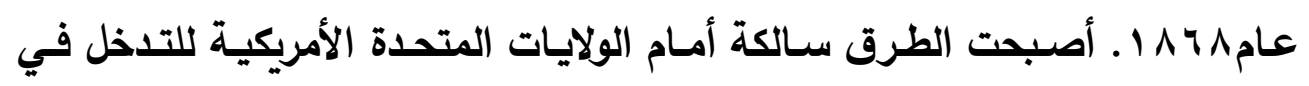
الشأن الكوبي المضطرب(')، لتحقيق أهداف عدة أبرزها(؟):

أولاً: حمايـة المصـالح الاقتصـادية الأمريكيـة والاسـتثمارات لاسـيما إنتـاج السـكر واستخراج المعادن مثل المنجنيز والفحم لإدامة عجلة الصناعات الأمريكية.

وثانياً: الاستحواذ على كوبـا وجعلها سـوقاً لتصريف البضـائع الأمريكيـة في كوبـا والاستفادة من موقعها الاستراتيجي المميز الذي ارتبطت أهميته بأهمية قناة بنمـا

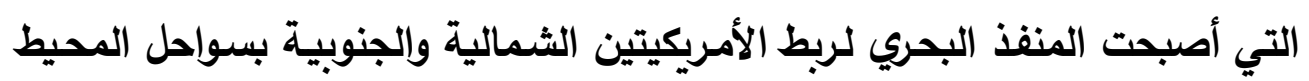
الهادي ومن ثم الثرق.

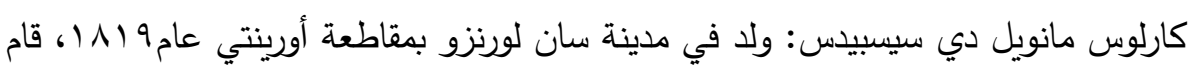

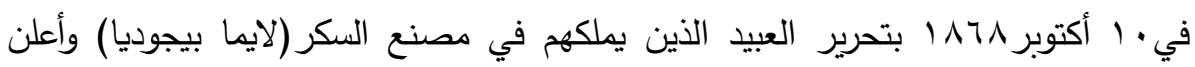

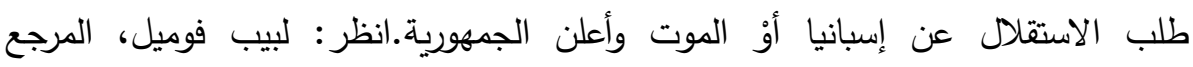

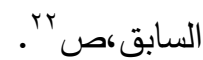

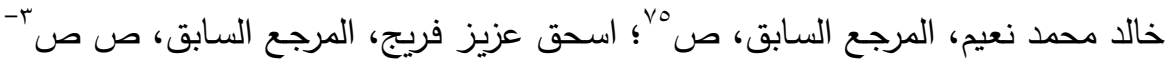

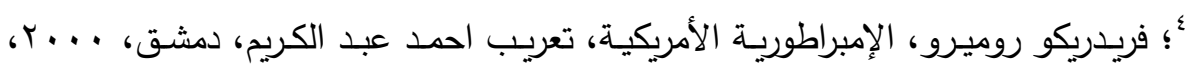
ص

Ferrer, Ada, Insurgent Cuba, Race, Nation, and Revolution, $८$ of North Carolina Press, Chapel Hill, 1999 , pp. ${ }^{r+r r}$; Aguilar, Luis E.,OP.,Cit., p. ${ }^{r o}$;

(r) Paterson, Thomas, American Foreign Relation. A history to $19 \mathrm{r} \cdot$, , (Boston r... , P. ${ }^{1 \leqslant r}$. 
وعلى هذا أوعز وزير الخارجية الأمريكي هاملتون فيش إلى الإدارة الأمريكية

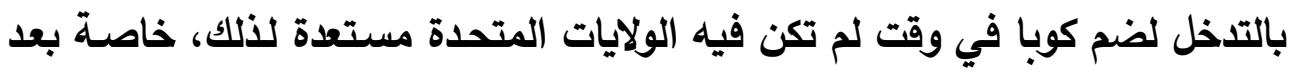

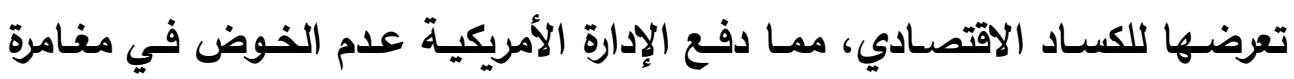

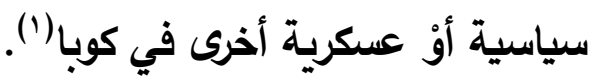

وإزاء تطور الأحداث الثعبية وتصاعدها في كوبـا أعلنت الإدارة الأمريكية في

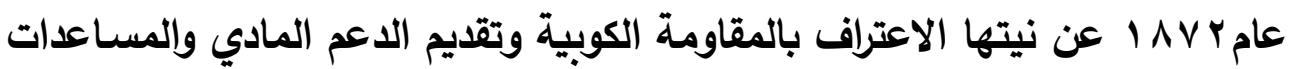

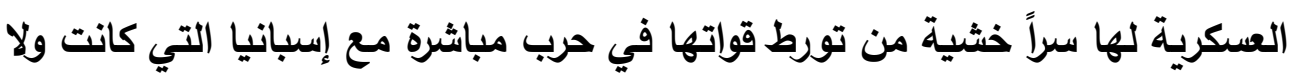

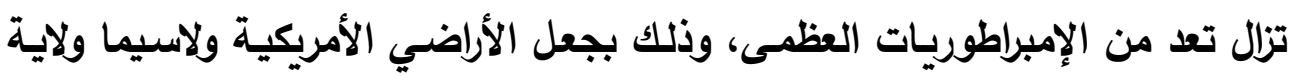

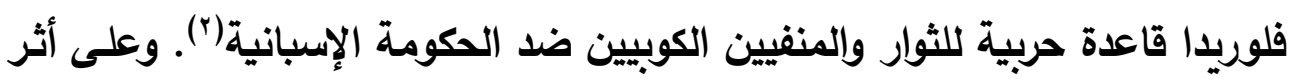

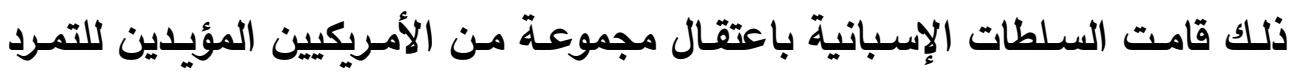

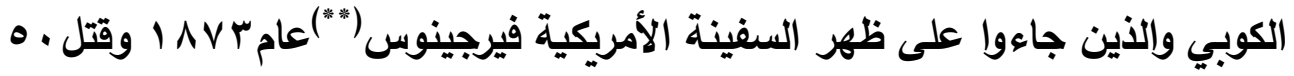
فرد منهم، فولات هذه الحادثة رد فعل عنيف في الولايات المتحدة الأمريكية وعداتها

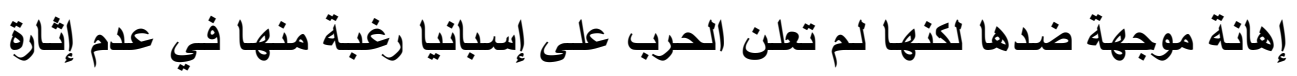

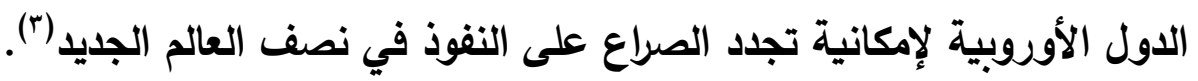
غير أن المقاومسة الكوبية سرعان مـا أخذت تلفظ أنفاسها الأخيرة بعد مقتل زعيمها المحامي كارلوس مانويل على يد القوات الاسبانية عام \& 1 1 ـ ـ وعلى الرغم

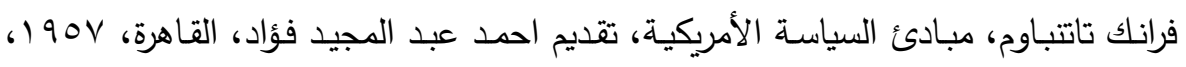

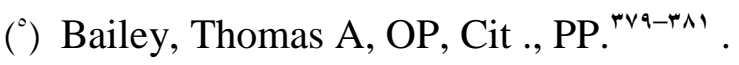

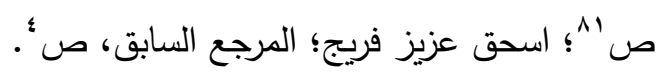

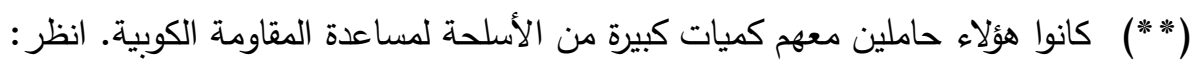
Faulkner, Herold wood, American Economic history New York, 190 \&, P. ${ }^{000}$.

(') Virginius, Encyclopedia Britannica, vol,, , op. Cit, p..$^{\wedge v v}$; Faulkner, Herold wood, OP.Cit., 190\%, P. ${ }^{000}$. 
José (") من تولي قيادة المقاومة الثعبية زعيم سياسي آخر وهو خوسيه مارتي Marti

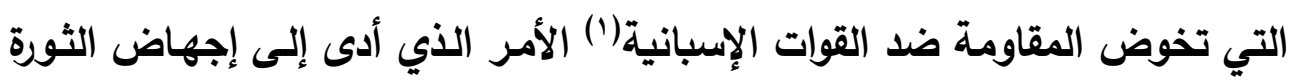
ولو مؤقتاً.

وقد تبـع ذلك قيـام الولايـات المتحدة الأمريكيـة بإبقـاء السـلطات الاسبانية الحاكمة في كوبا منشغلة بمواجهة الثوار الكوبيين الذين كانت تدعمهم اقتصادياً

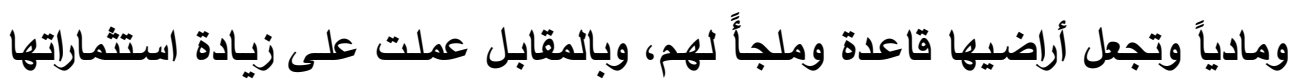

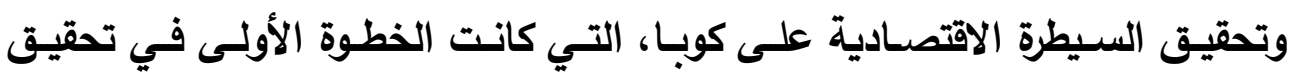
الاستعمار والاستحواذ العكري الأمريكي على كوبا مستقبلا (†).

وفى تسعينيات القرن التاسع عشر أخذت المقاومة الكوبية تنمو وتتطور على

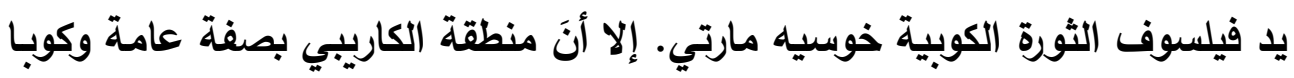

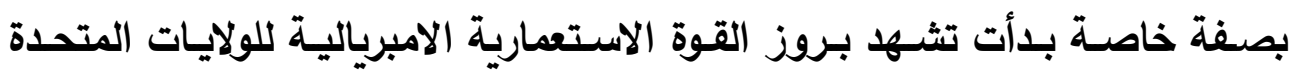

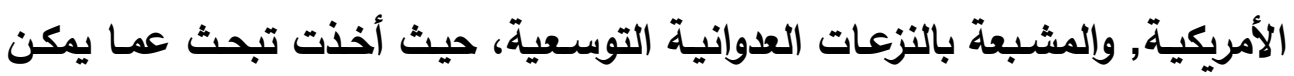

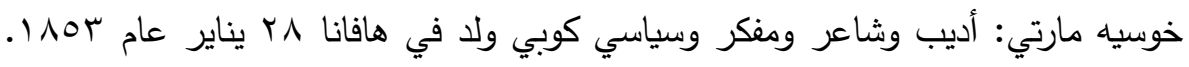

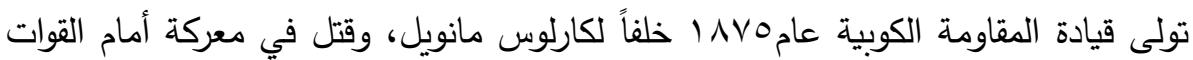

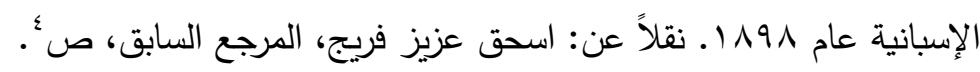

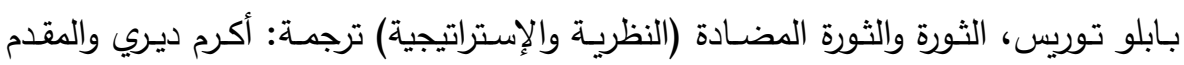

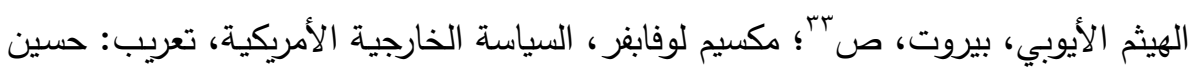

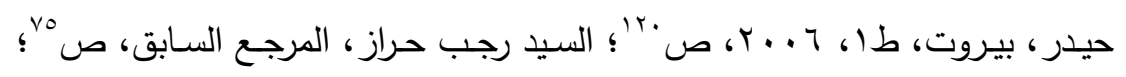
Bailey, Thomas A, OP. Cit ., P.

(') Paterson, Thomas, OP. Cit., P. ${ }^{19 r}$.

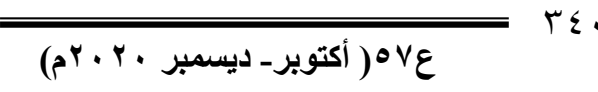
مجلة كلية الأداب- جامعة بني سويف 
20. (1)

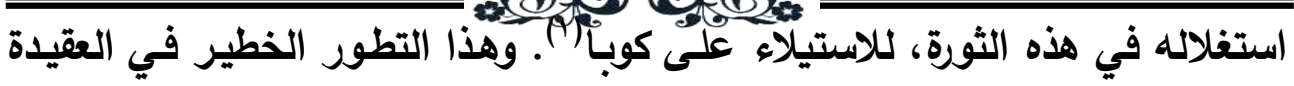
الأمريكية لم يأتِ من فراغ بل تمخض كنتيجة صريحة لاعتبارات عدة لعل أبرزها:

أولاً: التطور الصـاعي والازدهـار الاقتصـادي الذي حدث في البنيـة الاقتصـادية للولايات المتحدة خلال العقدين الثامن والتاسع من القرن التاسـع عشر، والذي غدا بحاجة ماسة إلى مصادر ومواد أولية لإدامته، وأسواقٍ لتصريف الفائض منده التهات

ثانياً: نمو حجم الاستثمارات الأمريكية وزيادتها في الخارج ومن ثم فهي أحوج مـا تكون لقوة عسكرية كبيرة للمحافظة عليها (†).

ثالثا: وجود بعض الساسة الأمريكيين البارزين الذين ينادون بتوسيع حدود الولايات

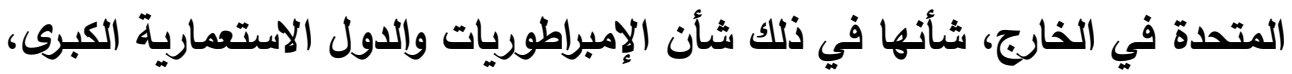

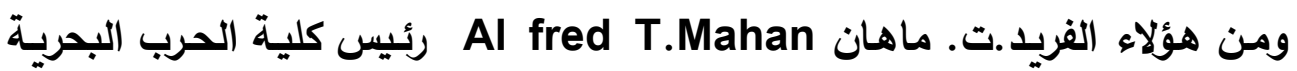

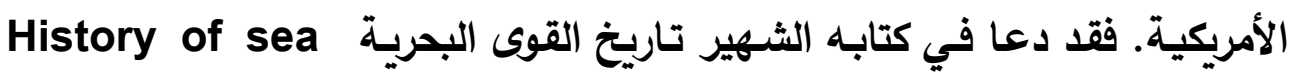

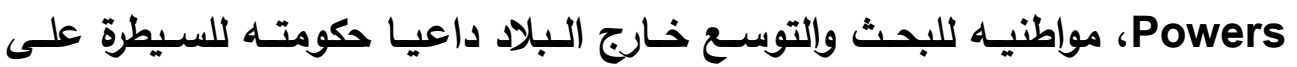
هاواي ومن ثم الفلبين(") موايهات.

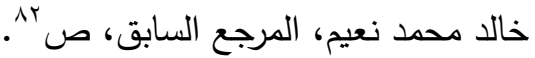

(॰) Mawinp, Bernstein, Foreign investments in Latin America, New York, 19\%., P. ${ }^{1 \leqslant \text {; }}$ Barbara, Tuchman W.,The Proud tower, Oxford University Press, New York, $197 \mathrm{Y}$, PP. ${ }^{19-10 .}$

(') Bailey, Thomas, OP. Cit, PP. ${ }^{100-1 v t .}$ 


\section{20}

رابعا: تأثر الإدارة الأمريكية ببروز بعض القوى الاستعمارية الجديدة مثل اليابان في

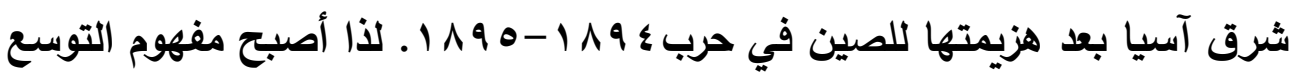
في الخارج هو ضرورة قوية من ضرورات بقاء البلاد وازدهارها (1). على كل حال لقد أدى تجدد الصراع الثوري في كوبـا عام ه9 11 إلـى أن تتصدر كوبـا نقاثـات الكونجرس الأمريكي. هذه الجزيرة التي كانت محور اهتمام الهام

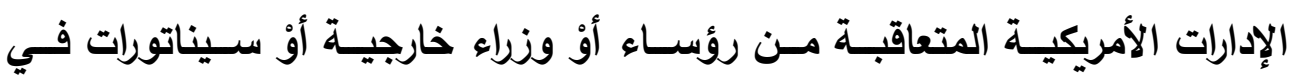

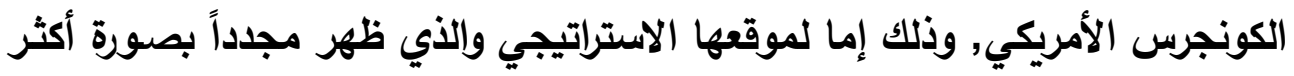

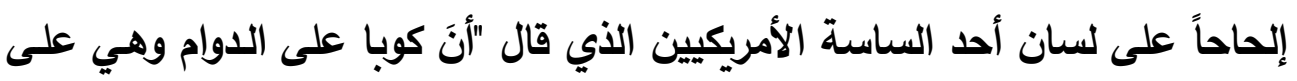
مرأى من شواطئنا وعلى وفق الاعتبارات كلها فقد غدت هدفاً لسياستنا الخارجية"((؟).

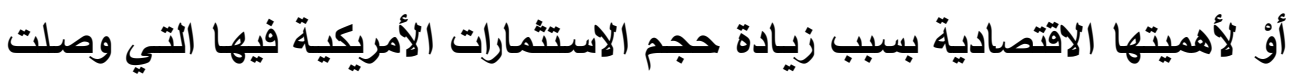

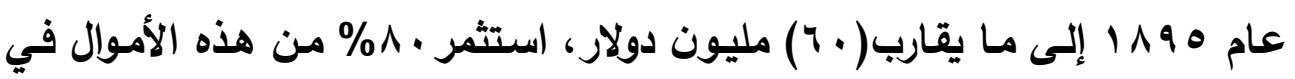

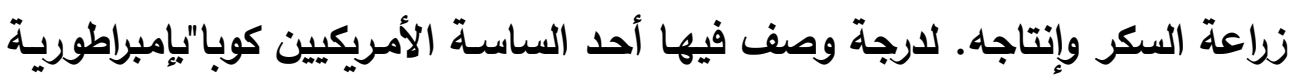

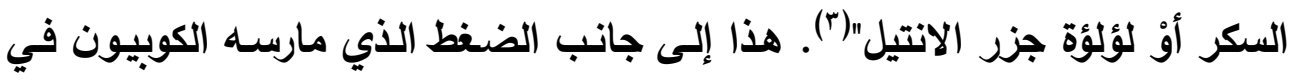

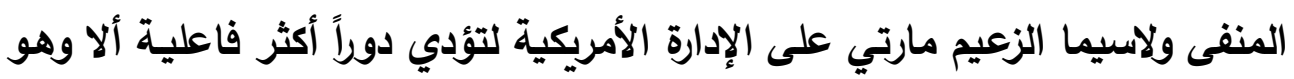

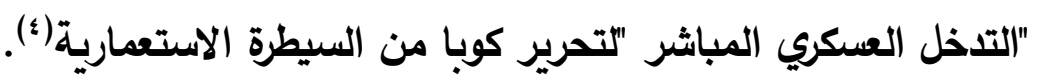
المحور الثاني: حادث تفجير البارجة ماين وإعلان الحرب على اسبانيا:

على أن أحداث عام 11 1 أدت إلى تغير حاسم في تاريخ الولايات المتحدة

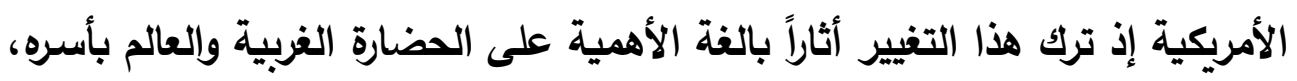

(') Barbara, W., OP. Cit ., PP. ${ }^{14 r-1 \text { i A }}$.

(") Paterson, Thomas, OP. Cit., P. ${ }^{19}$.

(\$) Bailey, Thomas A, OP. Cit., P. ${ }^{\text {} \wedge}$.

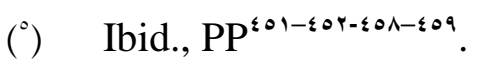

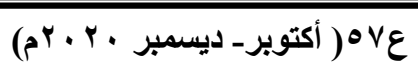

مجلة كلية الأداب- جامعة بني سويف 


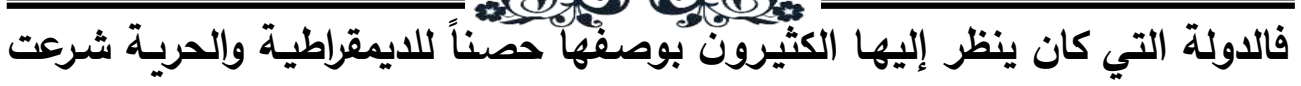

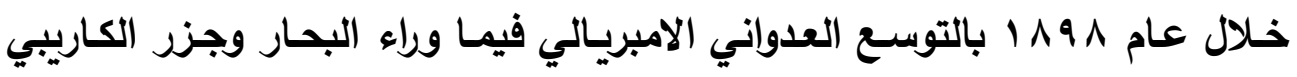

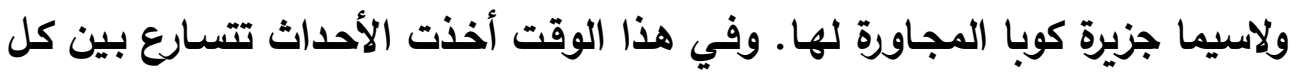

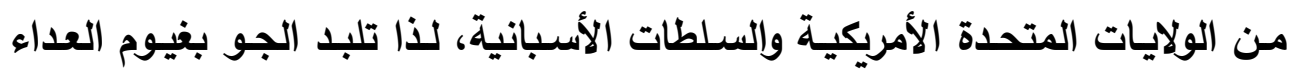
وأخذت الحرب بين الدولتين حول جزيرة كوبا تلوح في الأفق.

والحاصل إنه منذ عام ، 1199 كانت الولايات المتحدة الأمريكية تحتل مركز

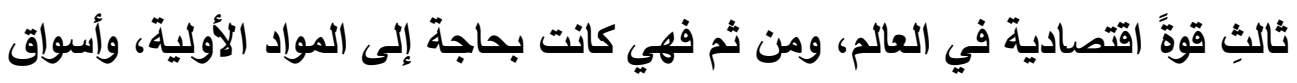

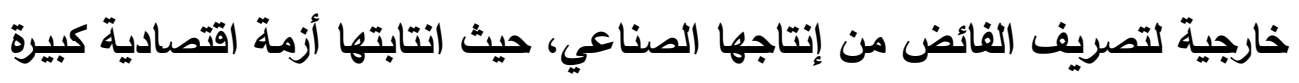

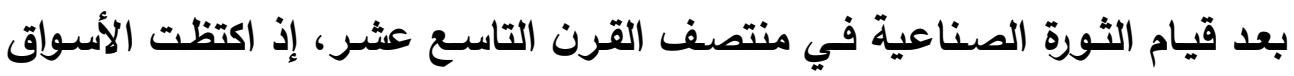

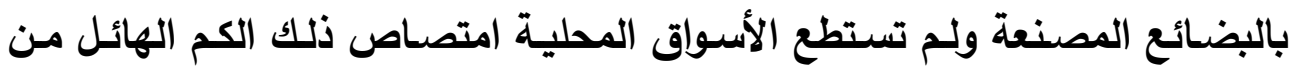

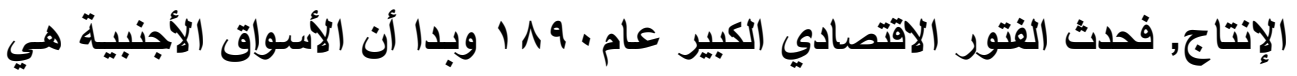

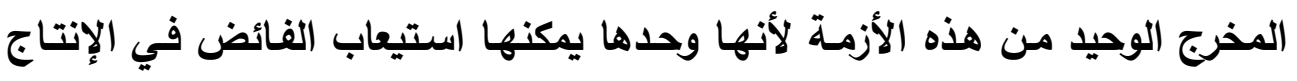

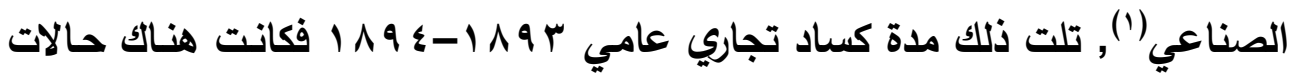

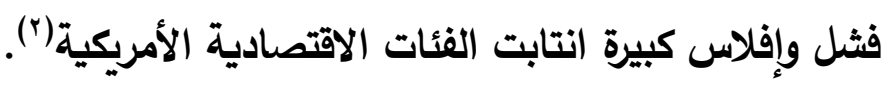
وهذه المنافسة والحاجة الاقتصادية هي التي حفزت الإدارة الأمريكية بالسعي

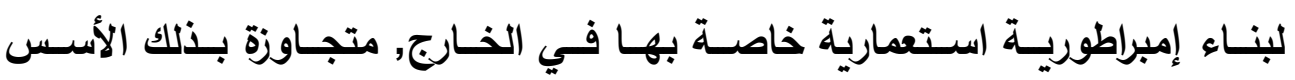

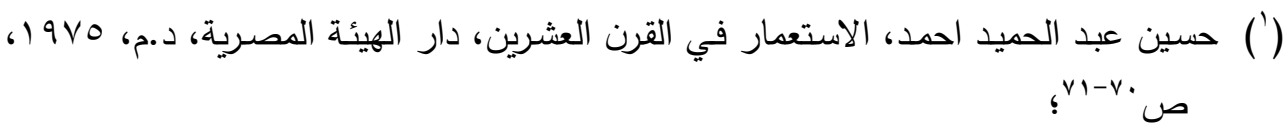

C.J. Bartlett ,The Global Conflict The Internationals Rivalry of the great

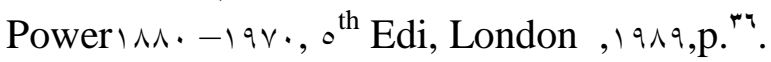

(') R. B.Mowat, M.A, Contemporary Europand Overseas 1 १९८-१९९., London, 190. , p. ${ }^{\text {r. }}$.

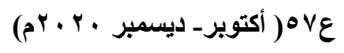
مجلة كلية الأداب- جامعة بني سويف 


\section{(4)}

الايمقراطية ومبادئ الحرية التي طوقت نفسها بها لقرابة قرن ونصف من الزمان ('). لذلك أخذت الولايات المتحدة تتحين الفرص لشن حرب على اسبانيا في ظل إنهاك المقاومة الكوبية للحكومة الاسبانية، موقنة أن إعلانها الحرب تحت ذربعة تحقيق استقلال كوبـا سيعزز موقعها ومكانتها في أمساكن أخرى من العالم، ولاسيما إنها كانت تخوض حرباً غير ناجحة ضد جبهة التحرير الوطني في الفلبين منذ منتصف

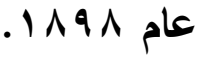

ولهذا اتخذت من حادثتين ذربعة أولها رسالة الوزير الاسباني المفوض في واشنطن انريكو ديبوي دي لومي Enrique De puy De Lome والتي بعث بها إلى أحد أصدقائه في كوبا والتي وضح فيها وجهة نظره في سياسـة ماكنلي خاصـة والسياسة الأمريكية بوجه عام(؟) وسرقت هذه الرسالة من مكتب بربد هافانا بواسطة أحد الكوبيين العاملين فيه وحصلت عليها جمعية الجوانتا Al Juanta الكوبية(")

(") Bailey, Thomas, OP. Cit, PP. ${ }^{100-1 \mathrm{vr}}$.

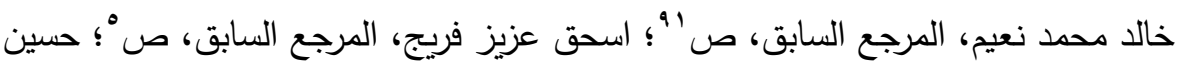

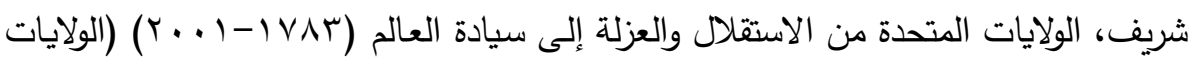

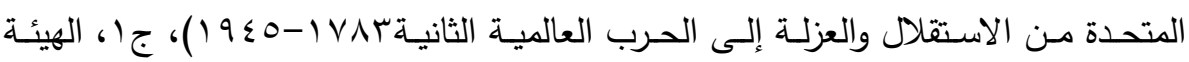

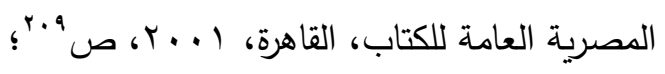

Sullivan, Mark, our times 19..-19ro, New York, 190r, P. ${ }^{\text {rq. }}$.; Commager, Henry Steel, Docu- ments of American History, Vol., , New york, $197 r$, p. ${ }^{4 r}$; Pérez-Cisneros, Enrique, En torno al "१८" Cubano, Editorial Verbum, Madrid, 199v, pp. ${ }^{1 \leqslant-1 \leqslant \leqslant . ~}$ جمعية جوانتا الكوبية، تعنى الجوانتا في اللغة الإسبانية مجلس أوْ لجنـة أوْ مؤتمر ، ولم

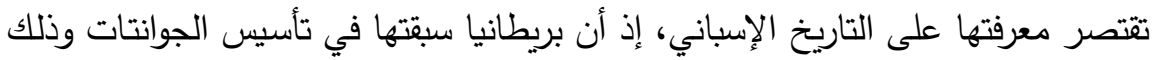

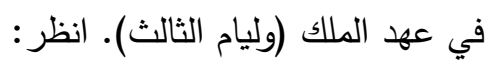


New York التي أرسلتها بدورها إلى رئيس تحرير صحيفة نيويورك تايمز

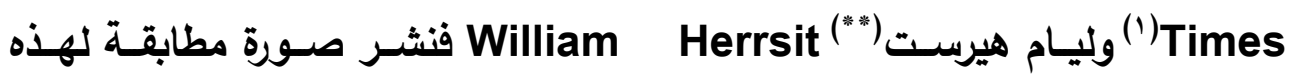

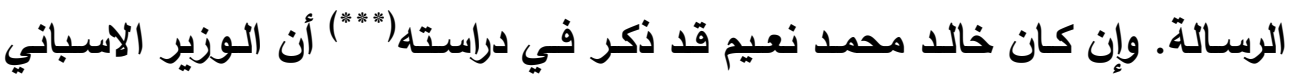
نفسـه هو من سلم الرسـالة لصحيفة هيرست ووصف الرئيس مـاكنليى فيها بأنـه يعمل على كسب إعجاب الجموع، وأنه مذنب لأنه يكن لأسبانيا سوء النية.

مهما يكن من أمر الرسالة فقد أدت إلى تأزم العلاقات بين إسبانيا والولايات

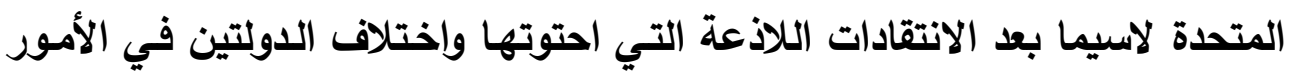
التجارية(؟) ومن جهتها حاولت إسبانيا تلافي الموقف بإقالة وزيرها إلا أن الرسبالة

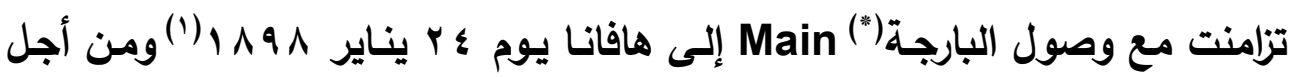

Al Juanta, The Encyclopedia Americana International, Vol: 14 , New York, I qvv, p. ${ }^{\text {ror }}$; Trasks, David F, Op. Cit., P.'.

جورج ف. كينان، الدبلوماسية الأمريكية، تعريب: عبدالله الملاح، دمشق، $9191 ،$ ص ص

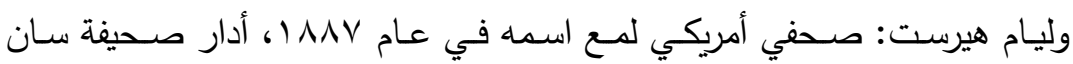

فرانسيسكو(examiner) ثم اشترى صحيفة (Morning Jurnal) في نيويورك. للمزيد

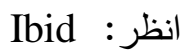

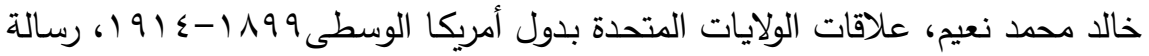

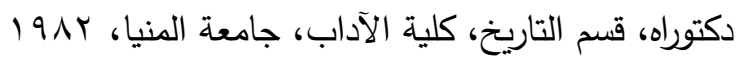

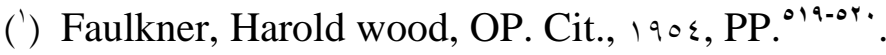

هذه البارجة التي كانت الإدارة الأمريكية قد أرسلتها لحماية كوبا وضمان سلامة المواطنين

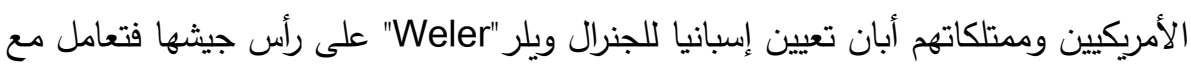
الكوبيين بوحشية على إثر تجدد الصراع بين الدقاومة الكوبية والسلطات الإسبانية، فشنت

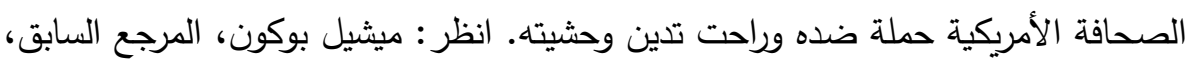




\section{0.}

إظهار حسن النية وافقت إسبانيا على زيـارة السفينة كوبـا، وتحت ظروف غامضـة

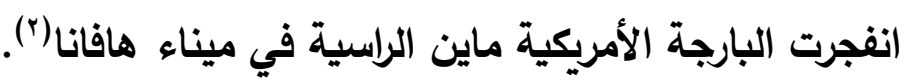

أودى هـا الانفجـار بحيـاة ـ ـ ب بــاراً مـن طـاقم البارجـة، فاتخـت الإدارة

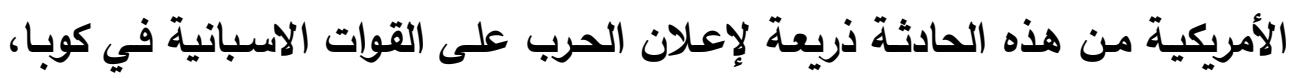

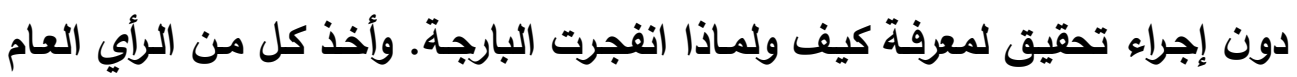

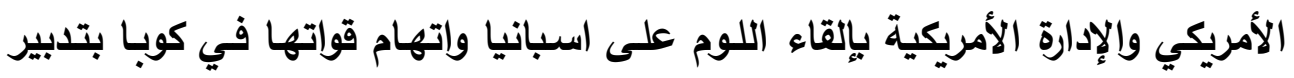

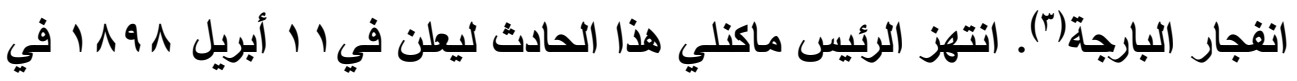

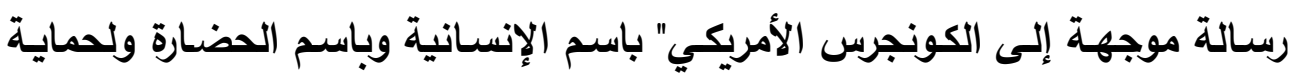
المصالح الأمريكية من الخطر ...لابد من إيقاف الحرب في كوبا"(أ).

والسؤال الذي يطرح نفسه هنا من كان وراء تفجير البارجة الأمريكية ماين؟

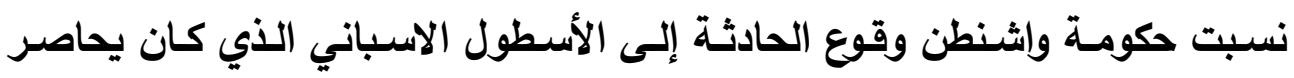

(') Could, Louis L, the Spanish-American war and president Mickinley, Knasas, $19 \wedge r, \mathrm{PP}^{r r-r s}$

فلاديمير خفوستون، الحرب الإسبانية الأمريكية، استيلاء الولايات المتحدة على كوبا

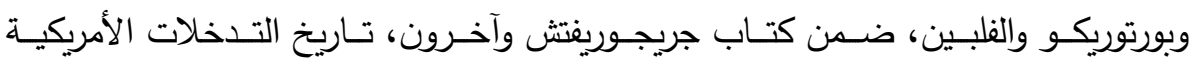

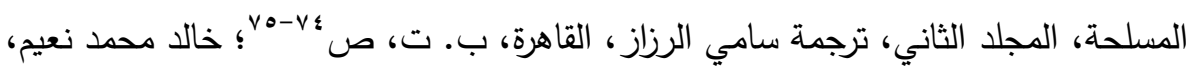

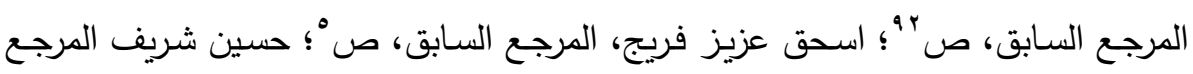

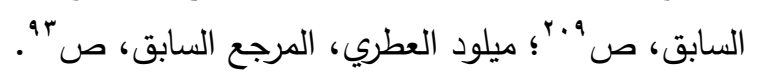

(') Paul, Jeffers H.,Colonel Roosevelt, Theodore Roosevelt goes to War

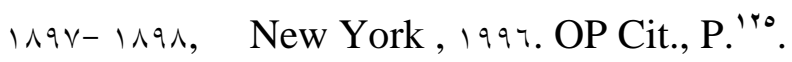

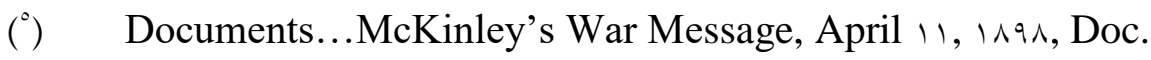

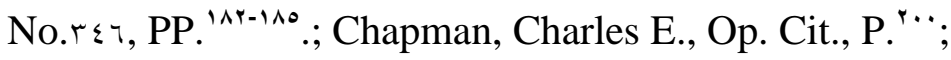

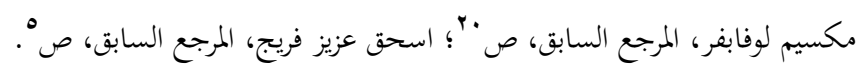

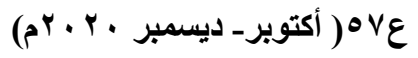

مجلة كلية الأداب- جامعة بني سويف 


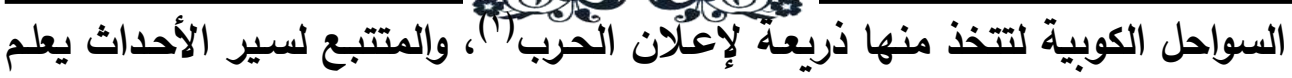

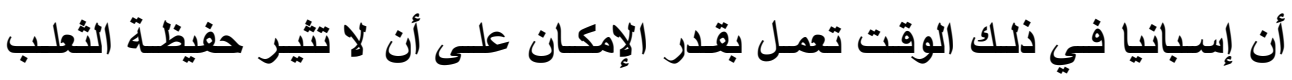

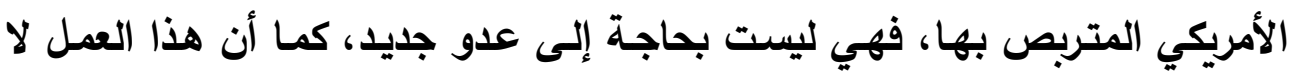

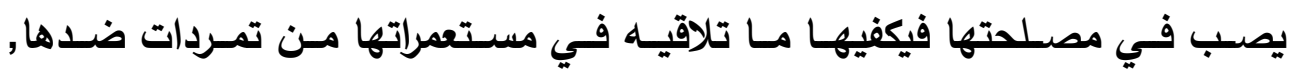

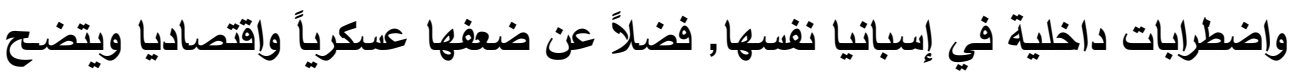

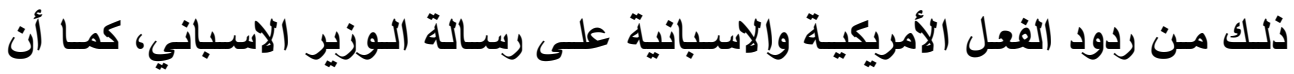
الوثائق لم تثبت أو تؤكد هذا الأمر.

ولكن أشارت بعض أصابع الاتهام إلى الثوار الكوبيين الذين أرادوا الحصول

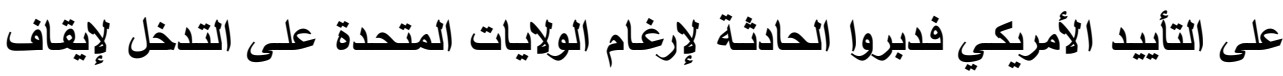
الحرب بينهم وبين السلطات الاسبانية وأن الولايات المتحدة على يقين بأن الثوات الثوار

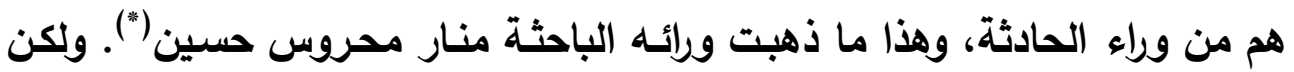
هذا الاتهام يبدو بإنه مخالف للمنطق والحقيقة وما تبين من التحقيقات.

ويبدو أن البحريـة الأمريكيـة هي المسئولة عن الحادثة بتدبير استخباراتي

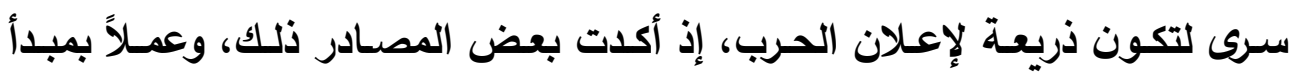

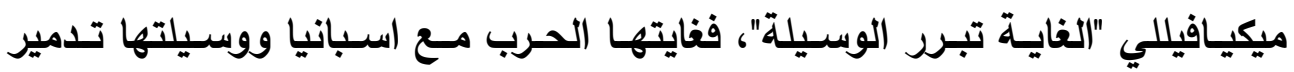

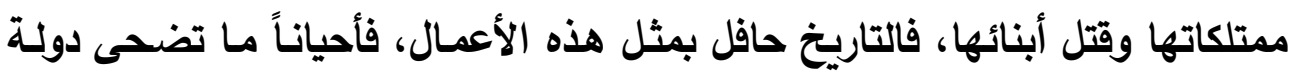

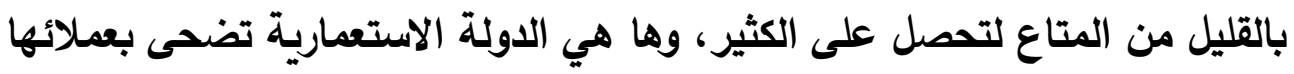

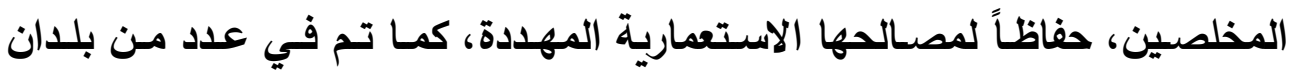
أمريكا اللاتينية والثرق الأوسط.

(") Bailey, Thomas A., op cit ,p. ${ }^{\text {sov. }}$

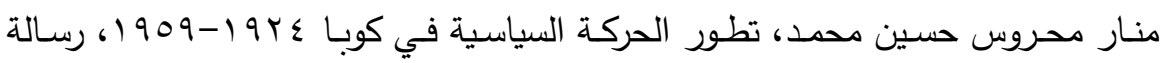

دكتوراه، قسم التاريخ، كلية الآداب، جامعة المنيا، (د.ت).

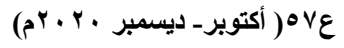

مجلة كلية الأداب- جامعة بني سويف 
وق تبين من التحقيقات التي أجرتها إسبانيا فور وقوع الحادث أن الانفجار سببه اشتعال في مستودعات الاخيرة نتيجة خلل داخلي في تصميم السفينة، وأنها

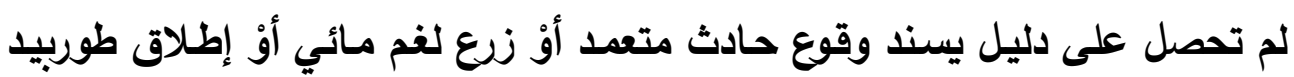

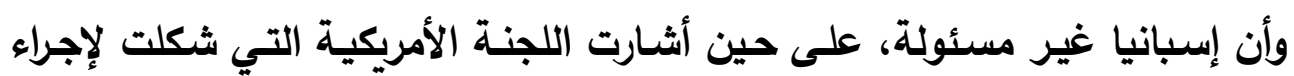

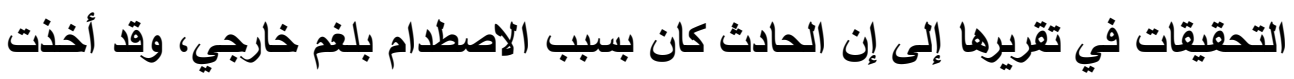

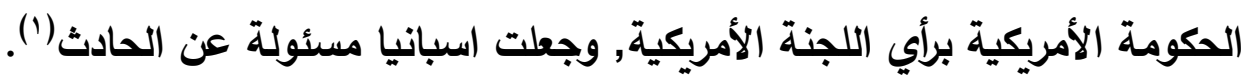

وفي اعتقـادي أن الولايـات المتحدة هي وراء الحسادث، نظراً لمـا جنته ومـا

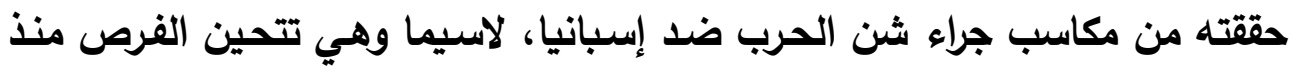

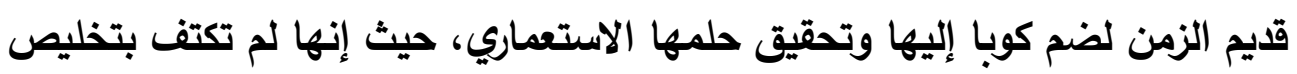

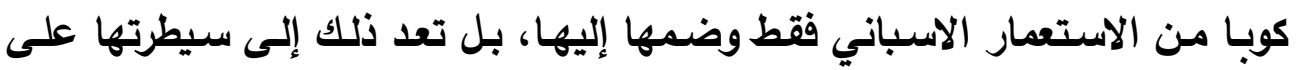
بورتوريكوPuertoRico وجوام Guam والفلبين Philippines.

وسواء كانت هذه الحادثة("*) من تدبير بعض الاسبانيين الذين لا يقدرون

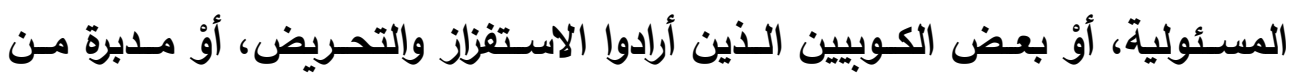

(') Ingham, Henry L, The Desting of a Democracy,vol.।,Washington, 19v^,p. ${ }^{\text {tro }}$; www. The Free Library.com

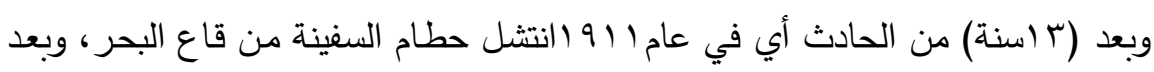

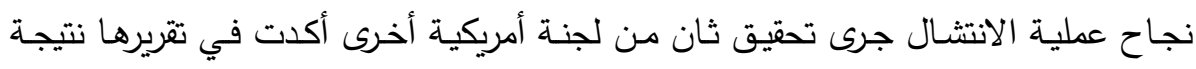

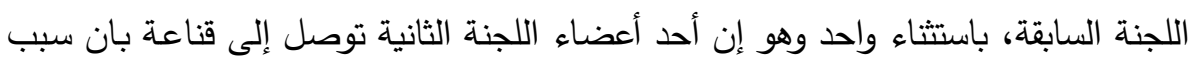

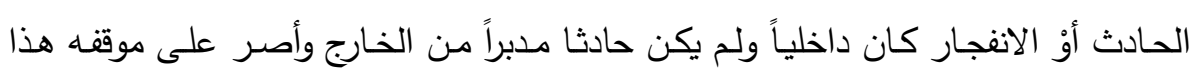

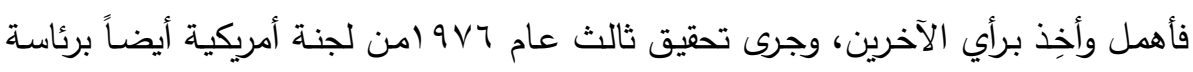

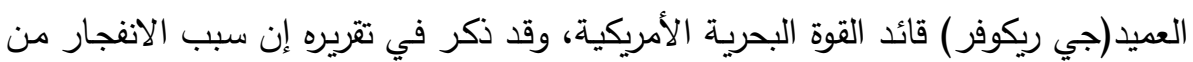

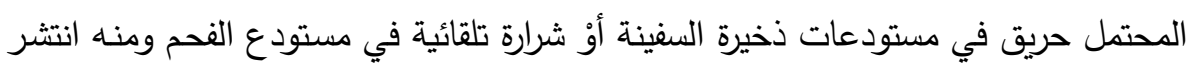

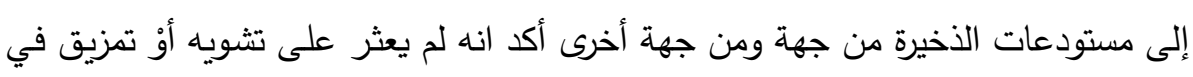

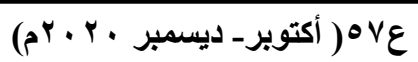

مجلة كلية الأداب- جامعة بني سويف 


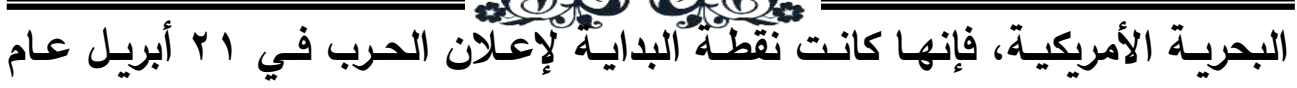

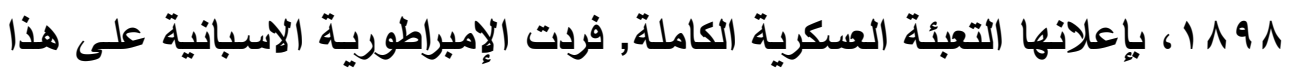
التصعيد العكري من حكومة وإشنطن ضدها بإعلانها الحرب على الولايات المتحدة

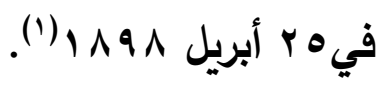

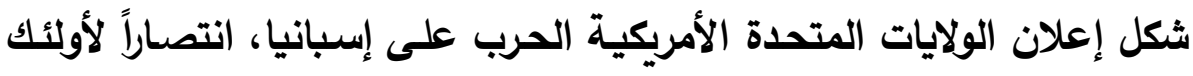

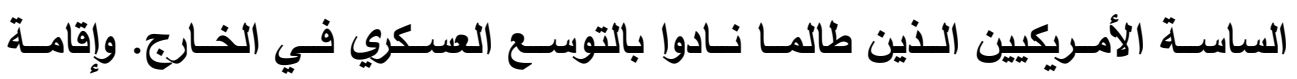

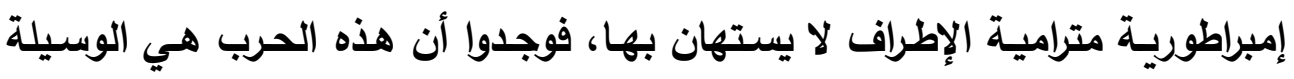
المثلى لتحقيق هذه الأهداف، والدليل على ذلك أن الإدارة الأمريكية بتأثير من هؤلاء كانت قد أعدت العدة بعد نهاية الحرب للحصول على بورتوريكو وجوام وإلفلبين(؟). ومن هذه الحادثة وغيرهـا من الحوادث نجد أن الولايات المتحدة الأمريكية

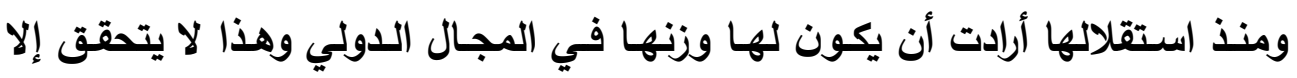

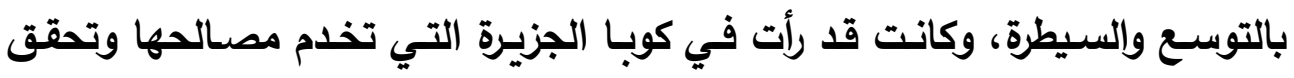

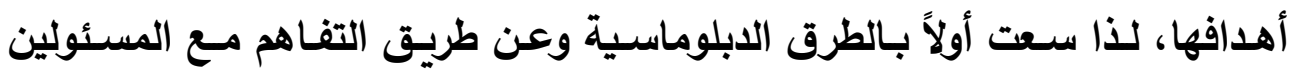

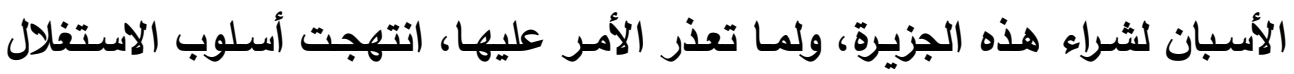

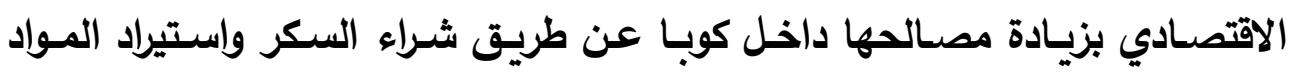

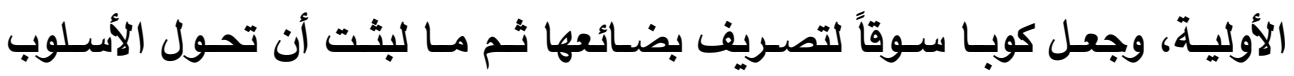

جسم السفينة الذي سيسببه انفجار لغم أو طوربيد اصطدم بها، وهذا ما يؤكد صحة تقرير

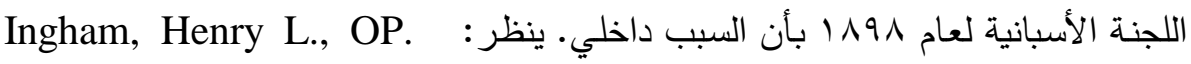

Cit.

(') Barbara, Tuchman W., OP. Cit, P. ${ }^{1}$; ; Paterson, Thomas, OP. Cit, P. ${ }^{r 14}$;

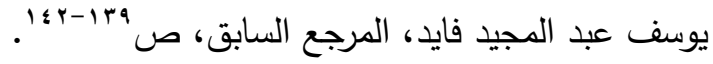

(') Charles, Dollar M., America: changing times-Since 1 ^ro, V. Two, New York, I qvv, $\mathrm{P}^{\mathrm{vir}}$. 


\section{2 (2)}

الابلوماسي إلى أسلوب آخر هو الاحتلال والسيطرة واستخدمت ذرائع واهية إلى حد

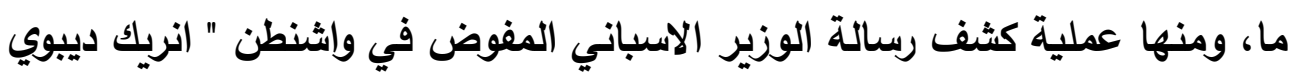

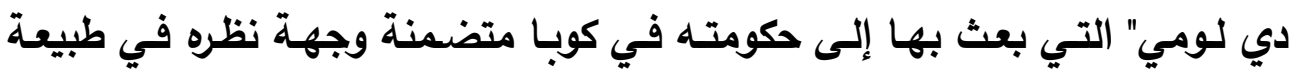
السياسة الأمريكية بوجه عام وفي سياسة الرئيس وليم ماكنلي بوجه خاص. لونها.

فبررت اسبانيا الأمر وأقالت وزيرهـا، وهذا كـاف لتقديم اعتذار لها، إلا أن

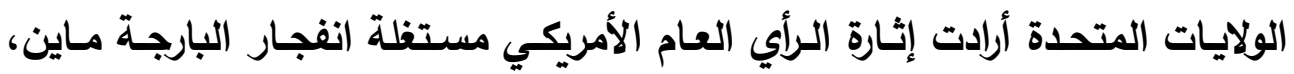

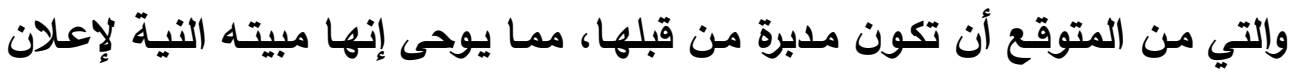

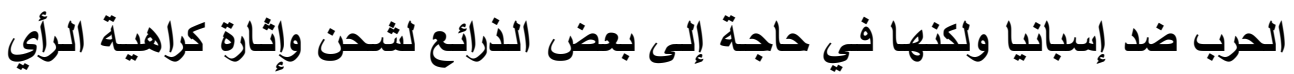

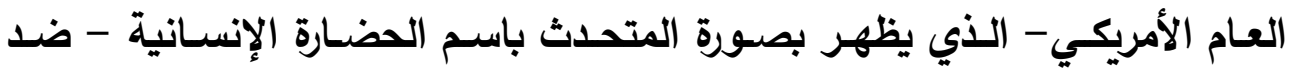

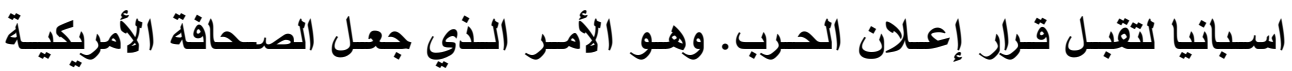

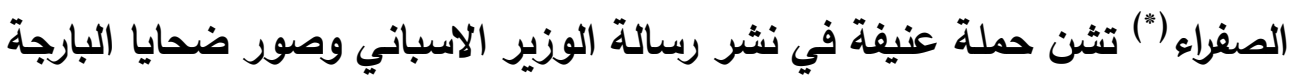
تحت عنوان مأساة البارجة.

فضلاً عن قصة الفتيات الكوبيات الثلاث التي نشرتها صحيفة هيرست التي

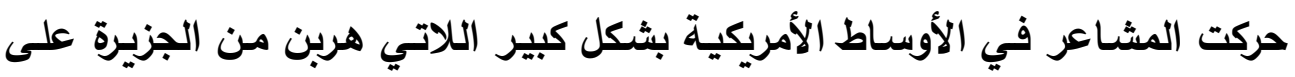

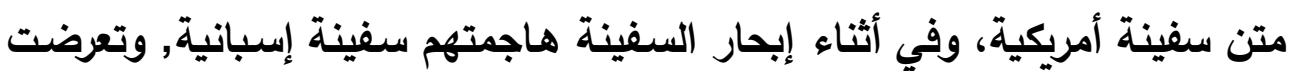

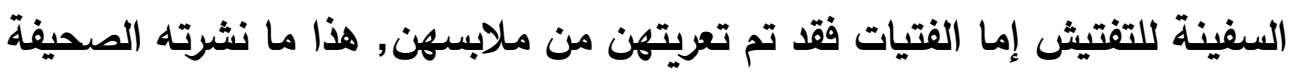

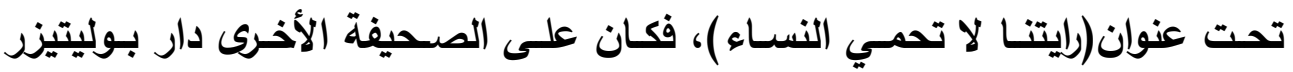

(") طرح هذا الدصطلح للتعبير عن صحافة الإثارة والتي من سبلها استخدام الأكاذيب ونشر الصور الفاضحة والمحرفة.

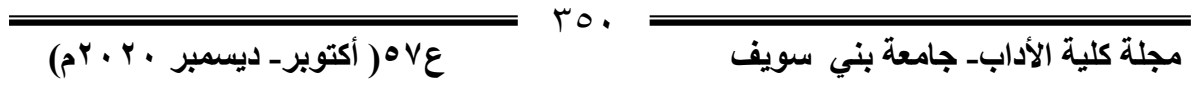




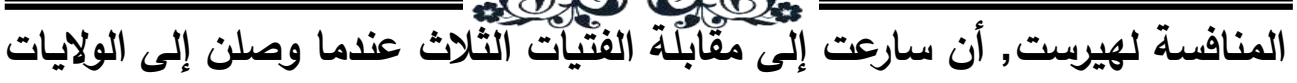

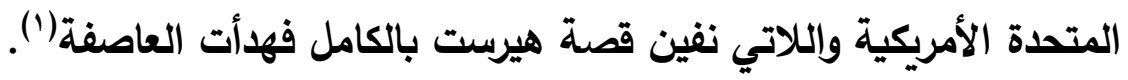

ولقد لعبت الصـف الأمريكيـة (الصـحافة الصفراء) دوراً مهمـاً في تـأجيج الأوضاع في كوبا ودفعها نحو التأزم بما تنشره من عناوين وروايات وقصص غير الهير

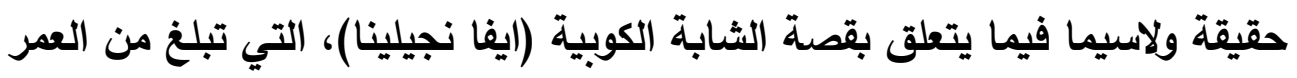

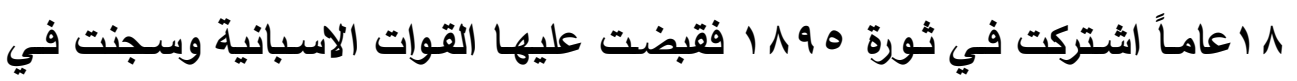

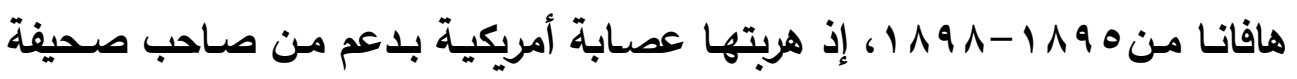

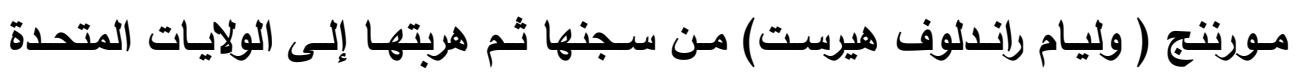

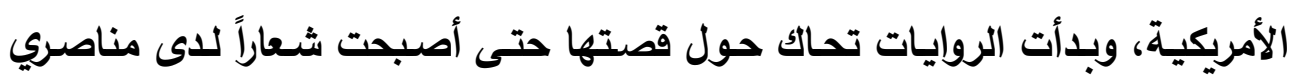

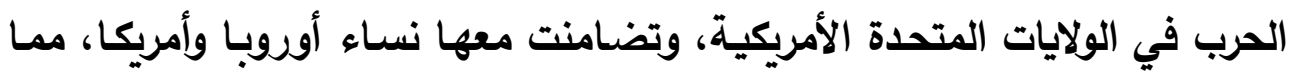

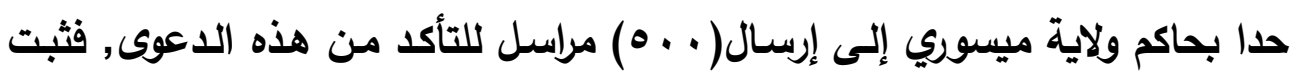

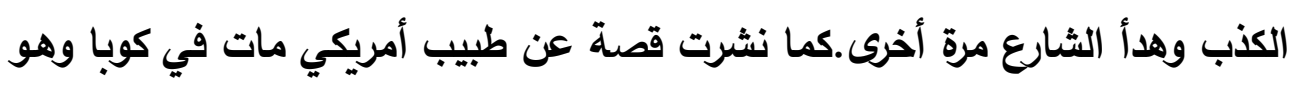
يمارس عمله الإنساني خلال مدة الثورة(†).

وكانت الصحافة الصفراء على عداء مع السلطات الاسبانية، لا سيما بعد أن

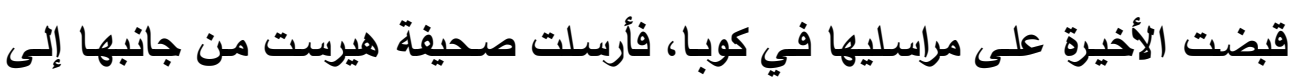

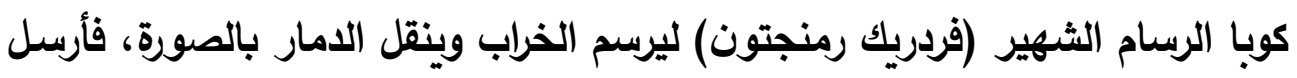

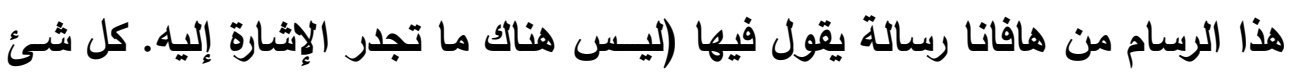

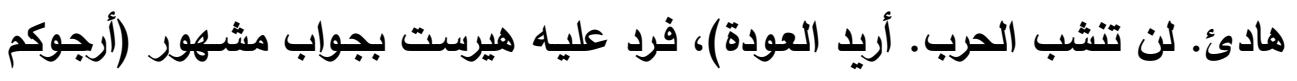

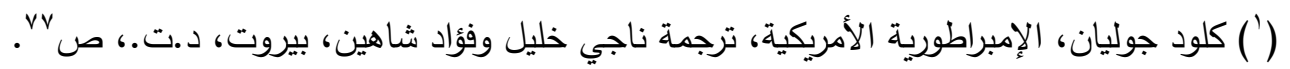

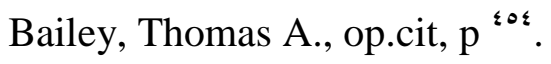

Wilder, Harold B. and other, This is

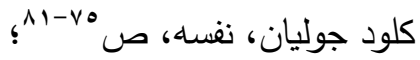
America`s story,Boston, 1907, P. $^{r} \stackrel{\text { ^ }}{\text {. }}$.

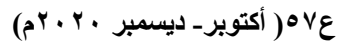
مجلة كلية الأداب- جامعة بني سويف 


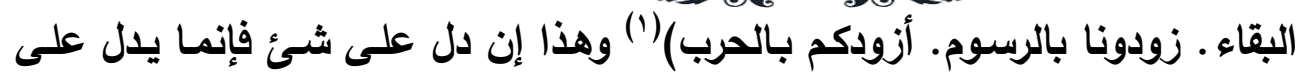
مدى النفوذ الذي تتمتع به الصحف الأمريكية.

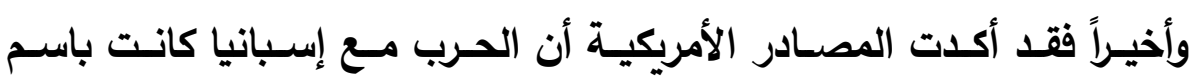

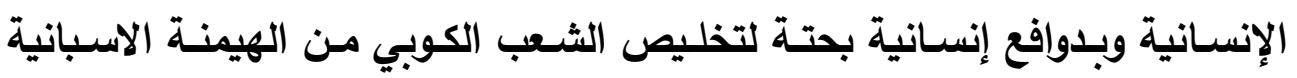

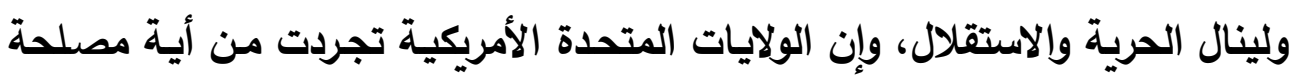

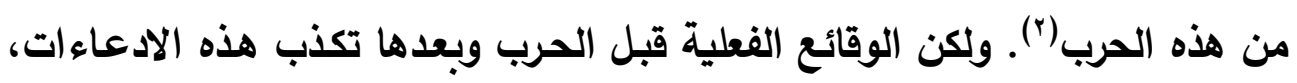

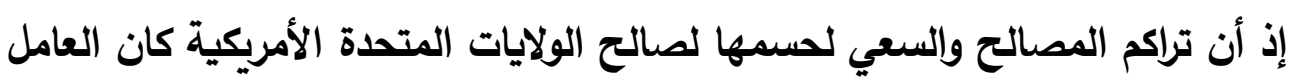
الحاسم في التخل في كوبا والهيمنة عليها.

الخاتمة:

عاثت كوبا انعكاساً للاوضاع المتردية للدولة المسيطرة (اسبانيا) في كافة

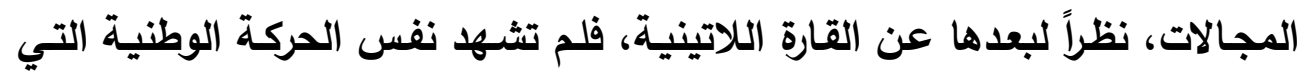

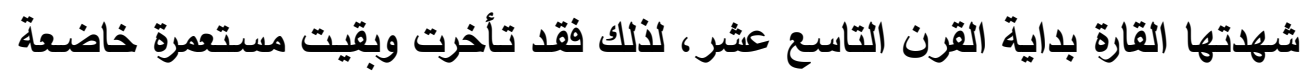

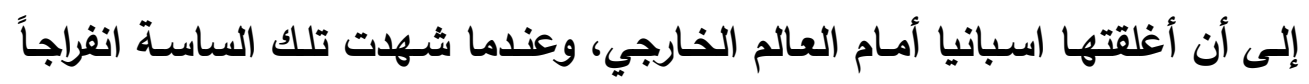

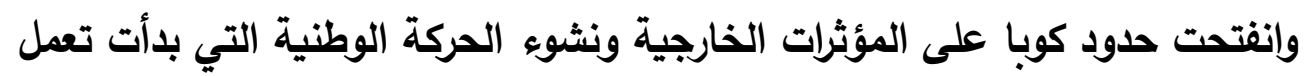

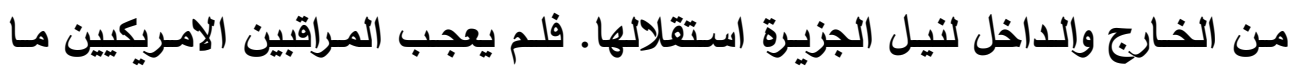

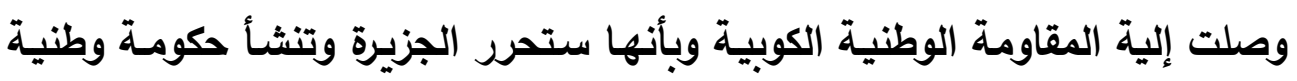
موالية لثعبها وذلك ما يعرض اهدافها السياسية في كل المنطقة الى الخطر كما

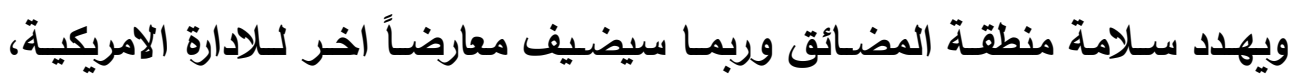

Faulkner, Harold wood,

$$
\text { نفسه، ص ص }
$$

American political, op.cit,P. ${ }^{019}$.

(") Pratt ,Julius W. ,America's Colonial Experiment ,New York , 190., p. ${ }^{2 r}$. 


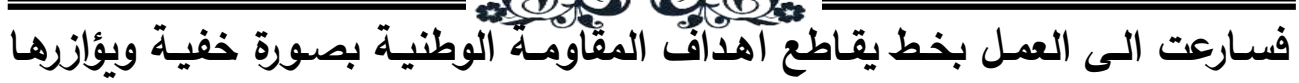

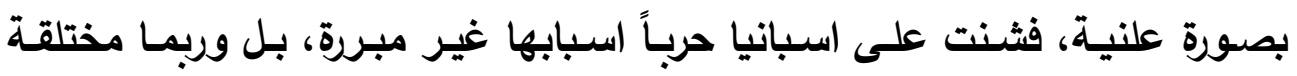

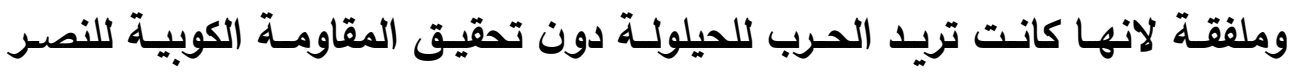

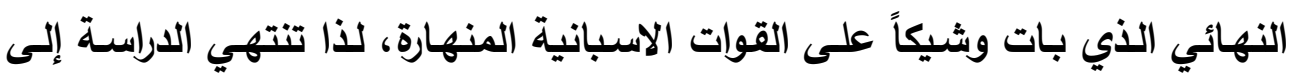

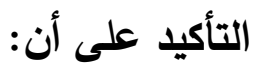

•• الولايات المتحدة كانت مبيته النية لاعلان الحرب منذ ان شارف الثوار على

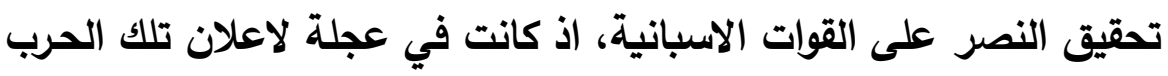

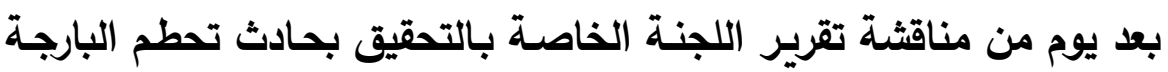

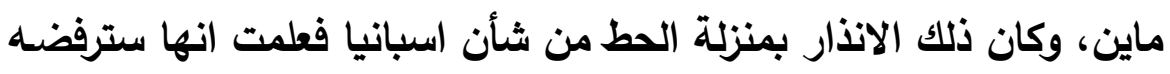

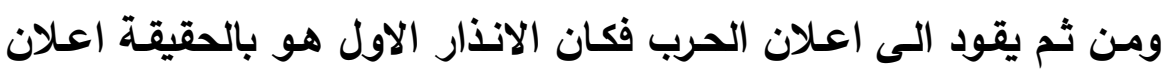
صريح بالحرب على اسبانيا. • • كان يمكن تجاوز الحرب لو ان الرئيس ماكنلي قدم الرسالة الاسبانية ومـا

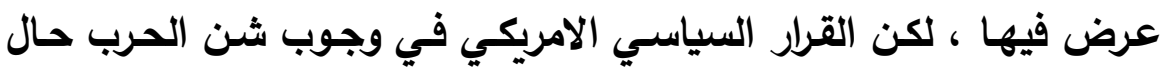

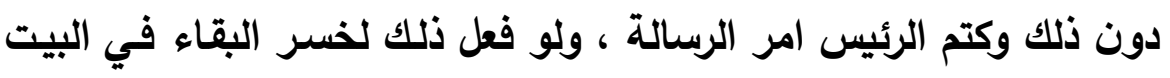

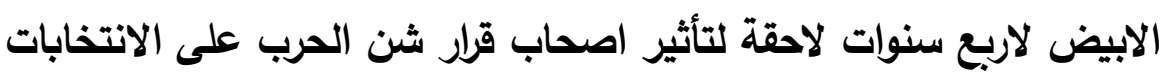

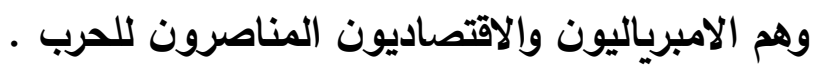

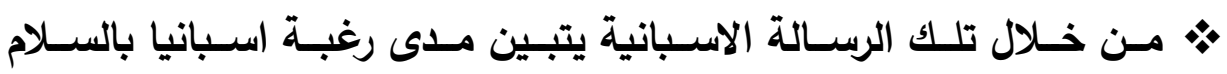

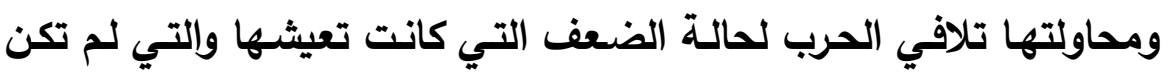
خافية على ملكتها وحكومتها مثلما لم تخف على الفيل الساسة الامريكيين. ويمكن الجزم بأن أساس كل السياسـة الامريكية التي مارستها في الكاريبي

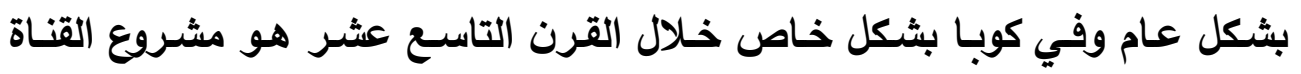
البرزخية التي تربط المحيط الهادي بالاطلسي فكانت كوبا بمثابة المكان الاستراتيجي هوني 
الذي يهيمن ويشرف على تلك القناة فكان لابـ للجزيره ان تتبع سياسـة تختارهـا الولايات المتحدة كخطوه أولى لتحقيق الهدف الرئيسي بالسيطرة على القناة، وعلى الحصول على قاعدة في الجزيـرة تـؤمن المحافظة على القنـاة كخطوة ثانيـة بعد

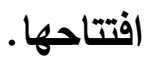

قائمة المصادر والمراجع: أولًا الوثائق : - 2 - أو

- - أمريكا اللاتينية والعالم العربي استعراض وتحليل، تقرير مرفوع الى جامعة الدول العربية

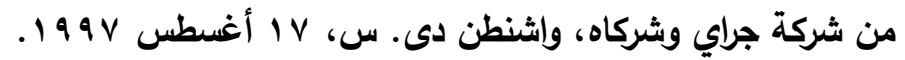

- Documents...McKinley's War Message, April 11, 1191 , Doc. No. $Y$ ร 4 .

ثانيًا: المراجع العربية والمترجمة:

- -

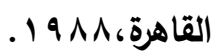

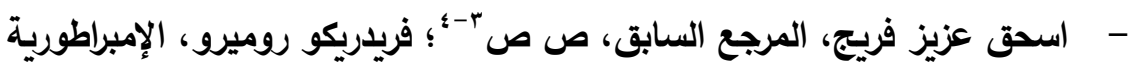

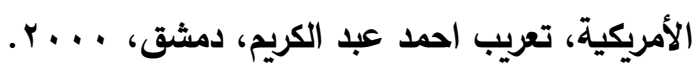

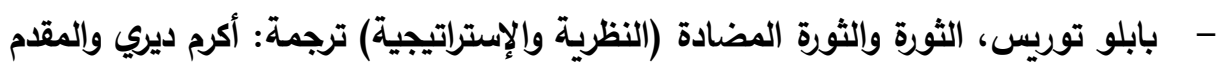
الهيثم الأيوبي، بيروت.

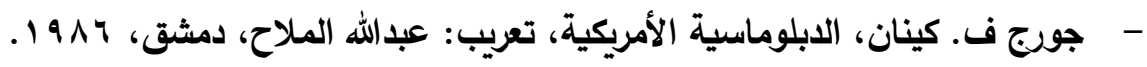

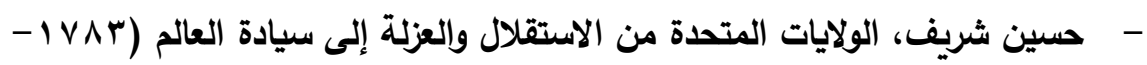

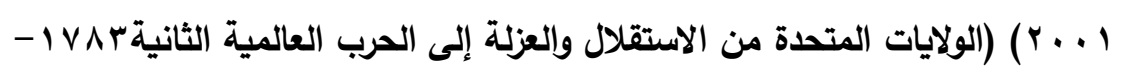

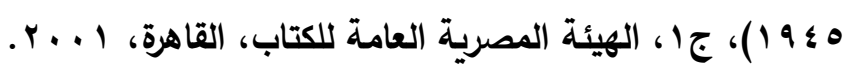

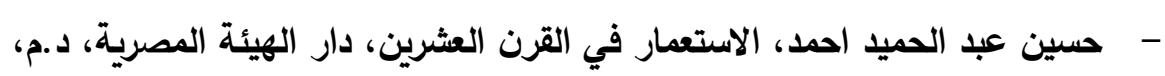
$.19 \vee 0$

مجلة كلية الأداب- جامعة بني سويف 


\subsection{0}

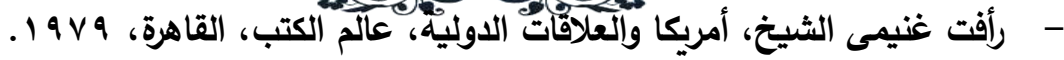

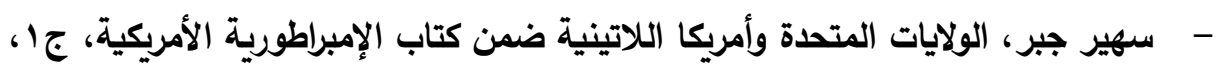

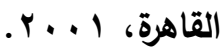

- شادي عبدالسلام، الوَيْلات المتحدة الأمريكية، طا، دار الطباعة المتميزة، القاهرة، $. r . . V$

- - فرانك تاتنباوم، مبادئ السياسة الأمريكية، تقديم احمد عبد المجيد فؤاد، القاهرة، $.190 \mathrm{~V}$

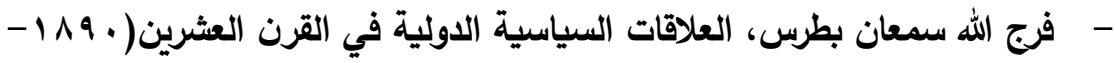

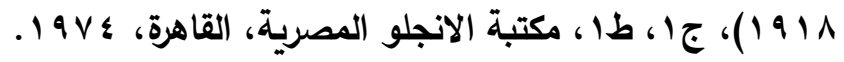

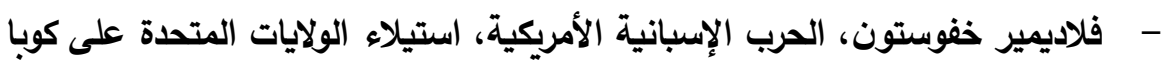
وبورتوريكو والثلبين، ضمن كتاب جريجوريفتش وآخرون، تاريخ التخخلات الأمريكية

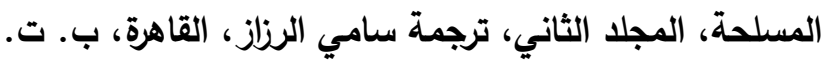

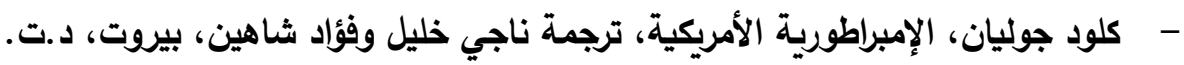
- ليب فوميل، كوبا للتمساح دموع حقيقية، دار الهلال،

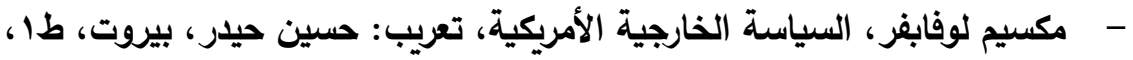

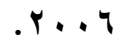

- ميشيل بوكون، أمريكا المستبدة "الولايات المتحدة وسياسة السيطرة على العالم"، ترجمة

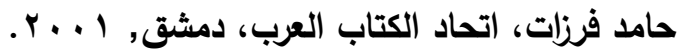

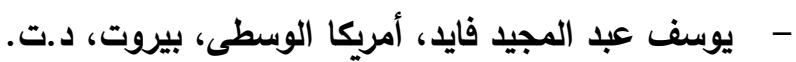

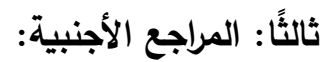

- ado, Roberto Regal, Latin American at the crossroads, New York, 199.

- Aguilar, Luis, Operation Zapata: The Ultrasensitiue Report and Testimoug of the board of Inquiry on the Bay of Pigs Aletheia Books, 1911. 


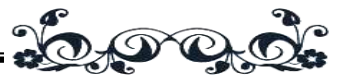

- Almaflor, Ada, Under the Royal plans: Childhoods in Cuba, London, $19 \wedge 1$.

- Bailey, Thomas A. A diplomatic history of the American people Englewood cliffs, New Jersey, $19 \vee \varepsilon$.

- Barbara, Tuchman W., The Proud tower, Oxford University Press, New York, 197v.

- C.J. Bartlett ,The Global Conflict The Internationals Rivalry of the great Power $\wedge \wedge \wedge .-19 \vee \cdot, 0^{\text {th }}$ Edi, London , $19 \wedge 9$.

- Chapman, Charles E.; A history of the Cuban Republic. A study in Hispanic American politics., New York, Octagon Books, 1979,Vol.I.

- Charles, Dollar M., America: changing times-Since $1 \wedge \uparrow \diamond, \mathrm{V}$. Two, New York, I qvv.

- Christopher, Abel and Torrents, Nissa, Eds Jose Marti: Revolutionary Democrat, London, $19 \wedge$ .

- Commager, Henry Steel, Docu- ments of American History, Vol.1, New york, 197r.

- Could, Louis L, the Spanish-American war and president Mickinley, Knasas, I $9 \wedge$ r.

- Faulkner, Herold wood, American Economic history New York, $190 \leq$.

- Ferrer, Ada, Insurgent Cuba, Race, Nation, and Revolution,

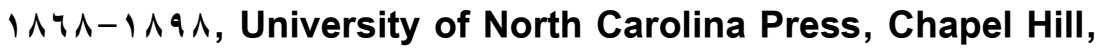
1999.

- Fontain, Roger W, on Negotiation with Cuba U.S.A American Enterprise institute for Public policy, Research, U.S.A, $19 \vee 0$. 
- Franklin, Jane, Cuba and The United States, Chronological History, sixth Printing, New York , Y.. .

- Ingham, Henry L, The Desting of a

Democracy,vol. I, Washington, $19 \vee \wedge$.

- Mawinp, Bernstein, Foreign investments in Latin America, New York, 197..

- Paterson, Thomas, American Foreign Relation. A history to 9 r., (Boston r...).

- Paul, Jeffers H.,Colonel Roosevelt, Theodore Roosevelt goes to War $\wedge$ $\wedge \vee-1 \wedge 9 \wedge, \quad$ New York, 1994.

- Pérez-Cisneros, Enrique, En torno al " $৭ \wedge$ " Cubano, Editorial Verbum, Madrid, $199 \mathrm{~V}$.

- Pratt ,Julius W. ,America's Colonial Experiment ,New York , $190 \cdot$.

- R. B.Mowat, M.A, Contemporary Europand Overseas $1 \wedge 9 \wedge-$ $194 .$, London, 190..

- Robinson, Albert G., Cuba old and New, Tower of Lafuerza, Havana, 1919.

- Sherry, Michael S., The Democratic Experience: A short American history (New York, 19^•).

- Sullivan, Mark, our times 19..-1940, New York, 190 r.

- Wilder, Harold B. and other, This is America's story, Boston, 1907.

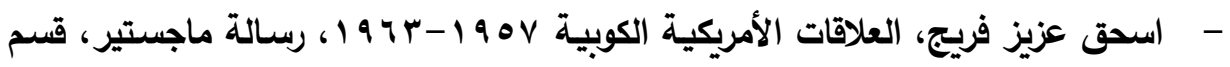

$$
\text { التاريخ، كلية الآداب، جامعة عين شمس، القاهرة، } 9 \text {. . . . . }
$$




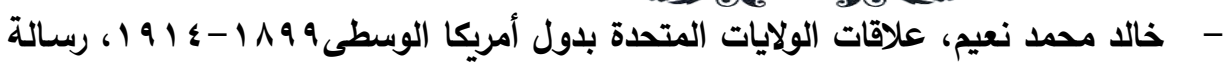

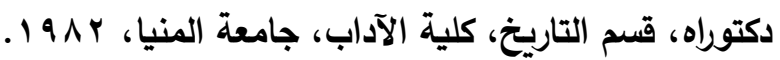

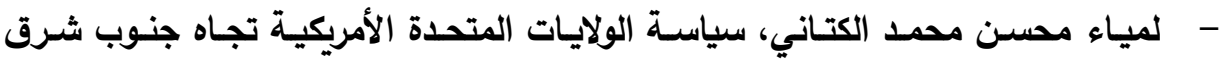

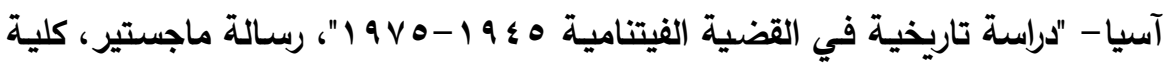

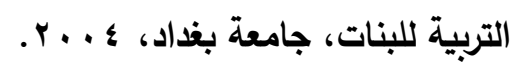

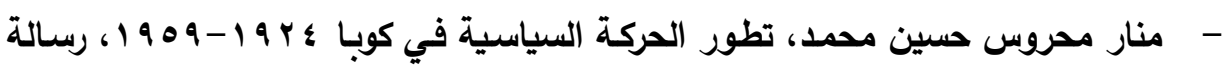

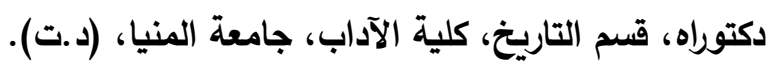

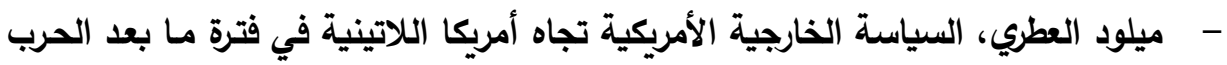

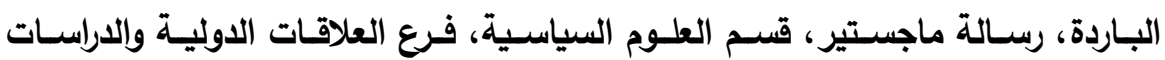
الإستراتيجية، كلية الحقوق، جامعة لخضر الحاج- باتنة، الجزائر، ^ . . r. خامسًا: الموسوعات الإجنبية:

- The New Encyclopedia Britannica, Chicago William, 19vr.

- The Encyclopedia Americana International, Vol: 17, New York, 19VV.

- مجلة السياسية الدولية، السنة الثانية، 1979 - 197 .

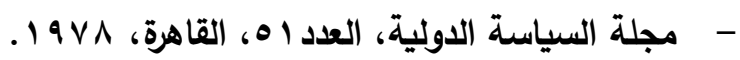
سابعًا: المواقع الإلكترونية:

- www.The Free Library.com. 